\title{
Preparation, Characterization, Laser Irradiation and Evaluation Biological Activity of Cobalt (II) with Mixed Ligands complexes
}

\author{
Ahlam Mohammed Y. Ahmed ${ }^{1 *}$, Zuhoor Fathi Dawoods ${ }^{2}$ \\ ${ }^{1 * 2}$ Department of Chemistry, College Education for Pure Science, University of Mosul, Mosul, Iraq \\ E-mail: ${ }^{1 *}$ ahlaam.chemistry2005@gmail.com, ${ }^{2}$ pd2faltaee@gmail.com
}

(Received September 08, 2020; Accepted October 21, 2020; Available online June 01, 2021)

DOI: $10.33899 /$ edusj.2020.128321.1110, (c) 2021, College of Education for Pure Science, University of Mosul.

This is an open access article under the CC BY 4.0 license (http://creativecommons.org/licenses/by/4.0/).

\begin{abstract}
New cobalt (II) complexes with mixed ligands including [(2- oxime-4-hydrazone) pentane $\left(\mathrm{L}_{1} \mathrm{H}\right)$ and 2-chlorobenzaldehyde thiosemicarbazone $\left(\mathrm{T}_{1} \mathrm{H}\right)$,or 3,4-dimethoxy acetophenone thiosemicarbazone $\left(\mathrm{T}_{2} \mathrm{H}\right)$; glycine $(\mathrm{GH})$ or metformin $(\mathrm{mf})$ ] have been prepared in both $\mathrm{pH}(6.5-7)$ and (9.5-10) using classical and microwave techniques. The resulting complexes have been characterized using physio- chemical and spectral techniques. The study suggested that the complexes have the generalformulate: $\left[\mathrm{Co}\left(\mathrm{L}_{1} \mathrm{H}\right)(\mathrm{TH})(\mathrm{BH})\right]\left(\mathrm{CH}_{3} \mathrm{COO}\right)_{2}$ at $\mathrm{pH}(6.5-7)$ and $\left[\operatorname{Co}\left(L_{1}\right)(T H)(G)\right] \operatorname{or}\left[\operatorname{Co}\left(L_{1}\right)(T)(m f)\right)$ at $(9.5-10)$, respectively $\left\{\right.$ where $\mathrm{TH}=\mathrm{T}_{1} \mathrm{H}$ or $\mathrm{T}_{2} \mathrm{H} ; \quad \mathrm{T}=$ deprotonated $\mathrm{T}_{1} \mathrm{H}$ or $\mathrm{T}_{2} \mathrm{H} ; \mathrm{L}_{1} \mathrm{H}=\mathrm{L}_{1}=$ deprotenated; $\mathrm{BH}=\mathrm{GH}$ or $\mathrm{mf} ; \mathrm{G}=$ deprotonated $\left.\mathrm{GH}\right]$. Hexacoordinated mononuclear complexes having distorted octahedral geometries have been investigated. No laser effects have been observed on solid ligands and complexes indicating that the compounds are stable and not affected by He-Ne laser (wave length are $=600-700 \mathrm{~nm}$ ). The biologicall activity of the ligands and complexes have been evaluated by agar plate diffusion technique against Echerichia coli, Klipsila pnnemoia, Staphylococcus aureus and Pseudomonas aeruginosa. Some compounds have been found to have antibacterial activity.
\end{abstract}

Keywords: Mixed ligands, Cobalt (II) complexes, thiosemicarbazone, metformin glycine.

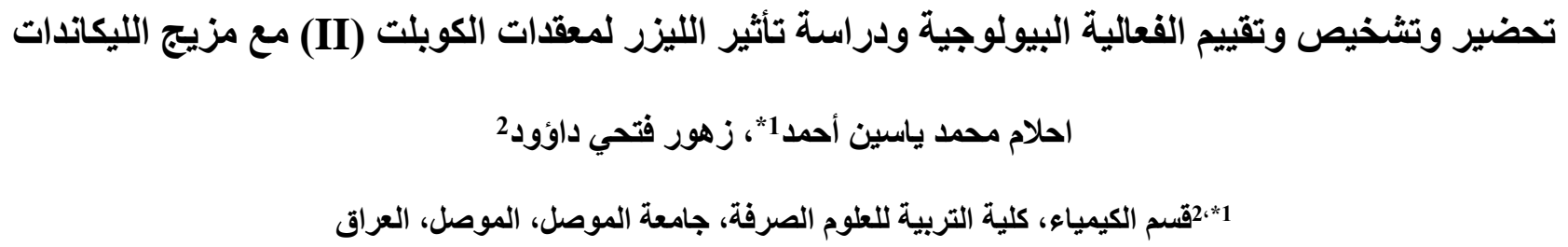




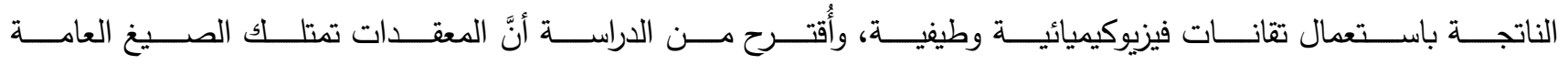

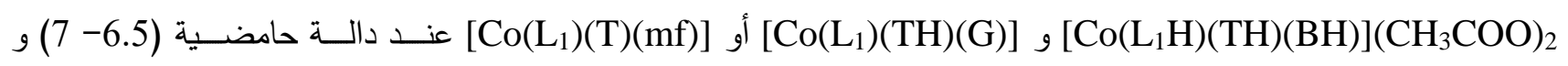

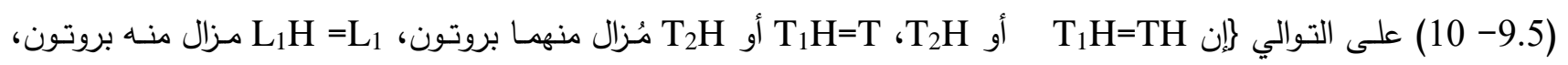

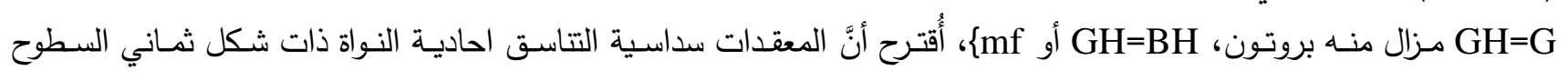

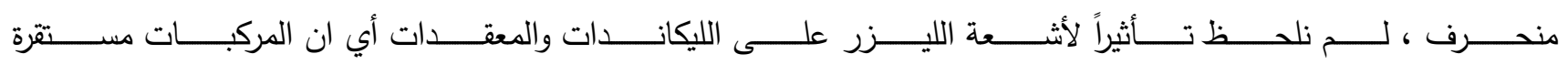

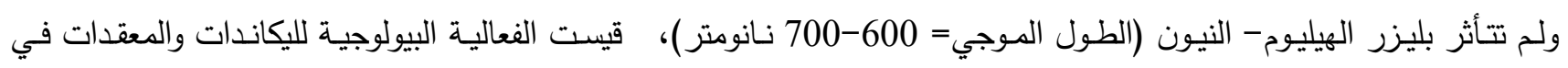
البكتريـا المرضـية Staphylococcus aureus و Mlipsila pnnemoia gscherichia.coli aeruginosa، بإستخدام تقانة الإنتشار على سطح الاكار، فلوحظ أنََّْ بعض المركبات تمتلك فعالية كمضادات بكتيرية. الكلمات الدالة: مزيج الليكندات، معقدات الكوبلت (II)، ثايوسيميكاربازون ، ميتفورمين، كلايسين.

\section{المقدمة}

إن الكوبلت (

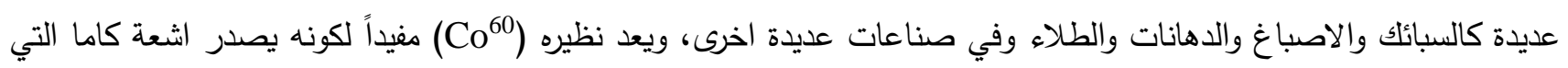

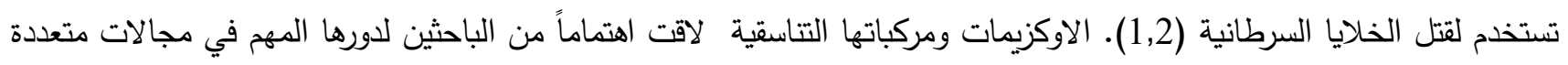
في المجالات الصناعية والطبية والحيوية فحضرت معقدات كثيرة ودرست خصائصها وتطبيقاتها (5,3). جذبت الهيدرازونات ومقعداتها الباحثين لتحضيرها ودراستها وتطبيقها في مجالات كثيرة نظراً لدورها البارز كمضادات حيوية ومضادات السل والسرطان

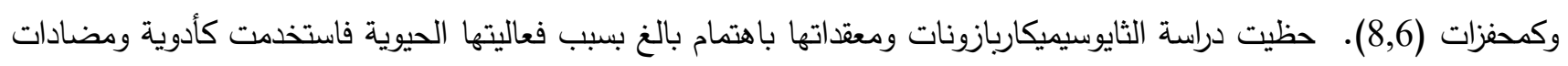
للملاريا وبعض السرطانات والسل والقرحة (12,9). أُستُخدم الميتفورمين كدواء خافض لسكر الدم النمط الثاني، و درس تأثيره واسلوب عمله، وهو مركب عضوي حضرت

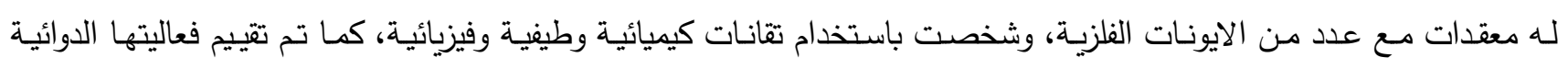

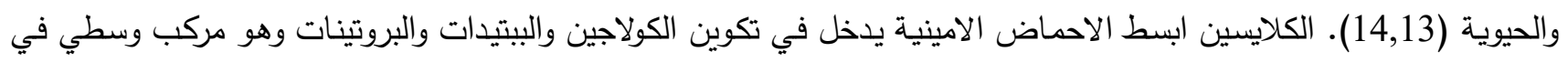
تحضير مختلف المركبات الكيميائية، ولقد درس تأثيره على بعض صفات النباتا يعد لليزرهيليوم-نيون(الطول الموجي= وسيلة لانتاج حزمة ضوئية، ولقد استخدت هذه التقانة في مجالات علمية وتطبيقية عديدة، وليزر الهيليوم- النيون (He-Ne) هو

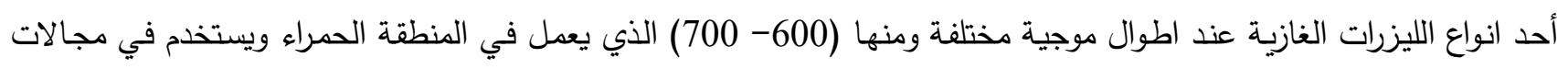
كثيرة (50-50).

، وحضر لله معقدات مع عدد من الايونات الفلزية ودرست خصائصها واستقراريتها وفعاليتها الحيوية (15). وقد ركز الباحثون

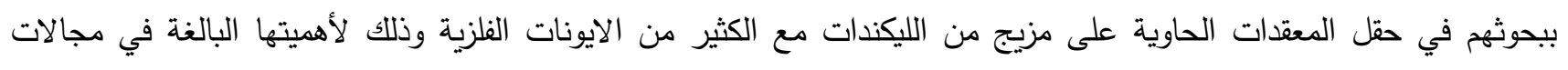
الحياة المختلفة، إذ لها دور مهم في العمليات الحيوية وكمضادات مكروبية، وفي المجالات الصناعية والصيدلانية...الخ $(19,16)$.

\section{الجزء العملي}

1- المواد الكيميائية الأولية: جُهزت المواد الكيميائية الاولية المستخدمة في البحث من قبل شركة Aldrich, Fluka, BDH, Alfa, Merck، وقد نقي 2، 4-دايون بنتان بعملية التقطير، كما تم تتقية الميتفورمين باعادة بلورته. 

حضر المركب الوسطي أولاً 2- اوكزيم-4- اون بنتان من تفاعل 2، 4 دايون بنتان مع الهيدروكسيل أمين هيدروكلوريد

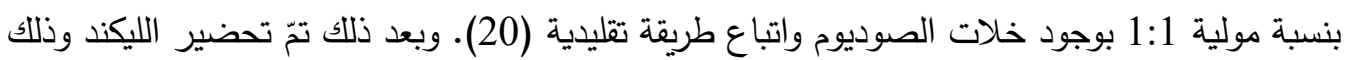

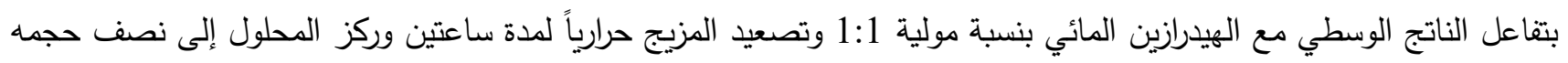

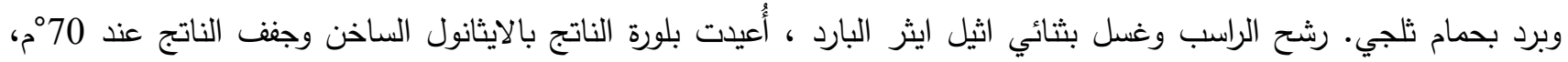
والمعادلتين أدناه توضح طريقة التحضير .

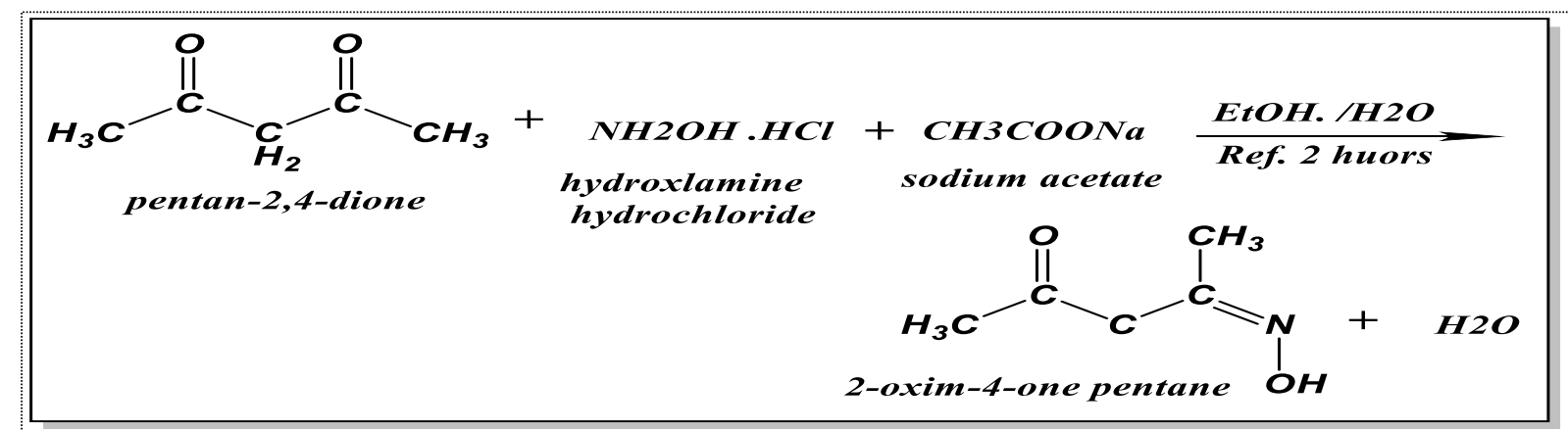

معادلة (1) تحضير المركب الوسطي

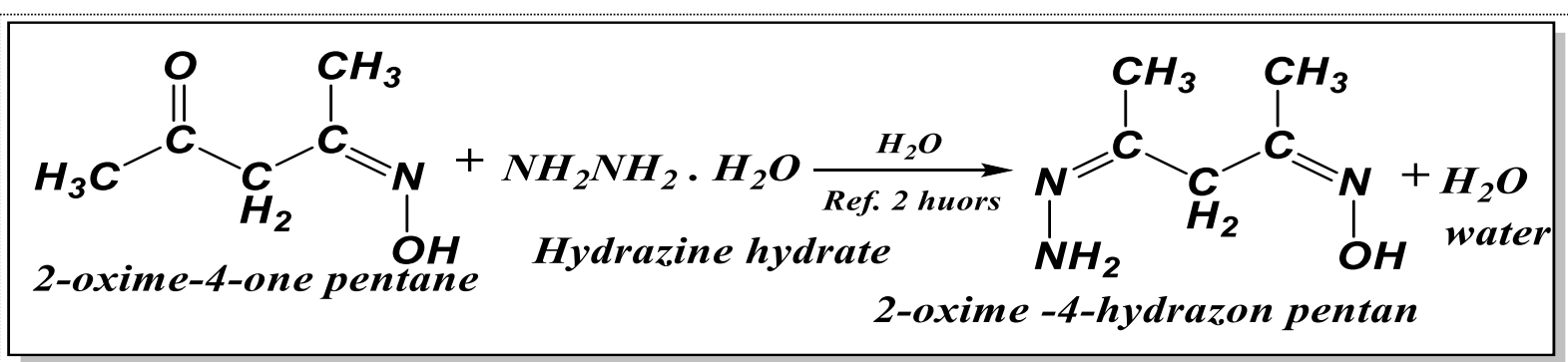

\section{معادلة (2) الليكند Le}

2.2 - تحضير ليكندي الثايوسميكاربازون:

حضر T و T T $T_{1} H$ من تفاعل 2-كلوروبنزالديهايد أو 3، 4-ثنائي ميثوكسي اسيتوفينون مع الثايوسميكاربازايد بنسبة مولية 1:1 وباتباع طريقة تقليدية (21). كما في المعادلة ادناه .

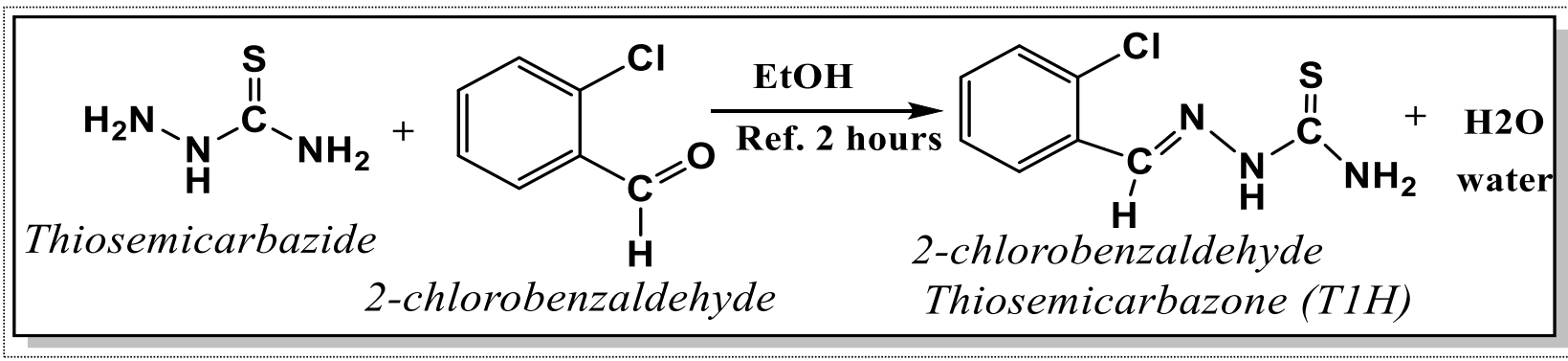

معادلة (3) تحضير الليكندات T1H 


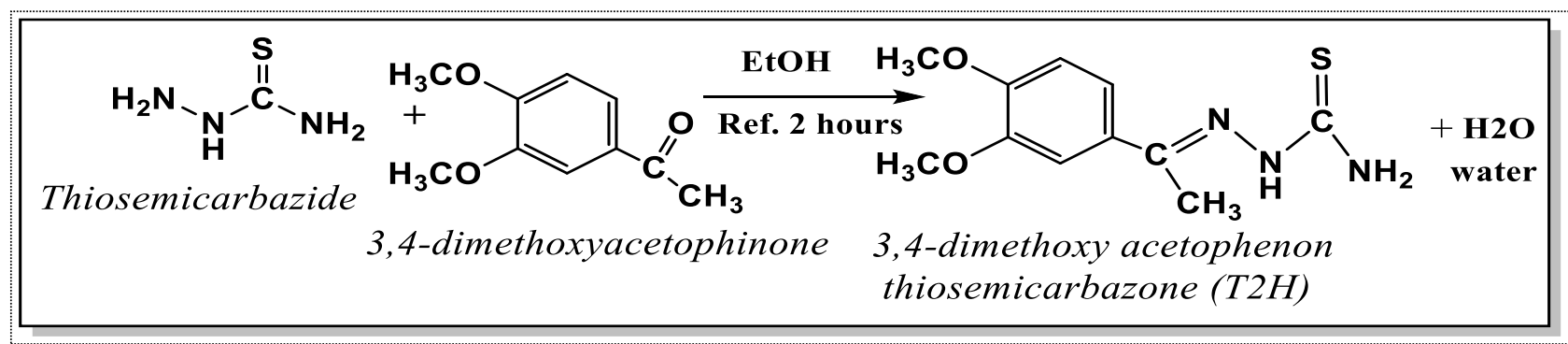

م2H معادلة (4) تحضير اليكند

والجدول التالي يبين الخصائص الفيزيائية لليكندات المحضرة .

\begin{tabular}{|c|c|c|c|c|c|}
\hline الليكندات & الكيميائيّة & 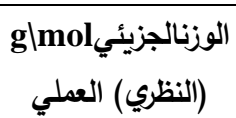 & اللون وطبيعة & $\begin{array}{c}\text { درجة الإنصهار } \\
\text { (م) }\end{array}$ & النسبة المئوية \\
\hline $\begin{array}{l}\text { 2-Chlorobenzaldehyde } \\
\text { thiosemicarbazone }\left(\mathrm{T}_{1} \mathrm{H}\right)\end{array}$ & $\mathrm{C}_{8} \mathrm{H}_{8} \mathrm{NSCl}$ & $213.6(214.7)$ & اصفر فاتح لماع & 198 & $\% 89$ \\
\hline $\begin{array}{l}\text { 3,4dimethoxyacetophenone } \\
\text { thiosemicarbazone }\left(\mathrm{T}_{2} \mathrm{H}\right)\end{array}$ & $\mathrm{C}_{11} \mathrm{H}_{15} \mathrm{~N}_{3} \mathrm{O}_{2} \mathrm{~S}$ & $253.3(253.3)$ & حليبي مسحوق & 201 & $\% 88$ \\
\hline $\begin{array}{l}\text { 2-Oxime -4- (3hydrazone) pentan } \\
\qquad\left(\mathrm{L}_{1} \mathrm{H}\right)\end{array}$ & $\mathrm{C}_{5} \mathrm{H}_{11} \mathrm{~N}_{3} \mathrm{O}$ & $129.1(129.2)$ & ابيض مسحوق & 348 & $\% 84$ \\
\hline Metformine (mf) & $\mathrm{C}_{4} \mathrm{H}_{11} \mathrm{~N}_{5}$ & (129.6) & ابيض مسحوق & 226 & - \\
\hline Glycine (GH) & $\mathrm{C}_{2} \mathrm{H}_{5} \mathrm{NO}_{2}$ & $(75.5)$ & ابيض مسحوق & 280 & - \\
\hline
\end{tabular}

3- تحضير معقدات الكوبلت (II)

حضرت معقدات الكوبلت (II) بطريقة التصعيد الحراري وفي كل من الوسطين المتعادل والقاعدي كما مبين ادناه :

1.3- تحضير المعقدات في الوسط المتعادل تمت مفاعلة خلات الكوبلت المائية مع ثلاث ليكندات عضوية بنسبة مولية

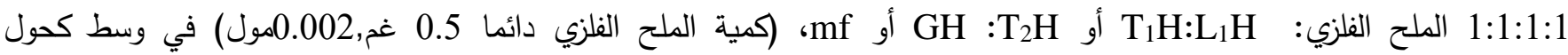
مائي(ايثانول,ماء)، صعد المزيج حرارياً لمدة ثلاث ساعات ، ثم ركز المحلول إلى نصف حجمه وبرد ورشح الراسب وغسل بثنائي اثيل

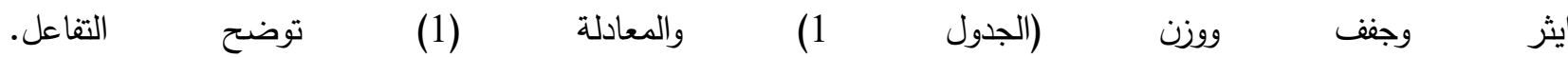
$\mathrm{Co}\left(\mathrm{CH}_{3} \mathrm{COO}\right)_{2} .4 \mathrm{H}_{2} \mathrm{O}+\mathrm{L}_{1} \mathrm{H}+\mathrm{TH}+\mathrm{BH} \rightarrow\left[\mathrm{Co}\left(\mathrm{L}_{1} \mathrm{H}\right)(\mathrm{TH})(\mathrm{BH})\right]\left(\mathrm{CH}_{3} \mathrm{COO}\right)_{2}+4 \mathrm{H}_{2} \mathrm{O}$

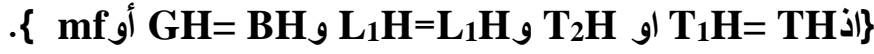

3.2- تحضير المعقدات في الوسط القاعدي: حضرت المعقدات بمزج محلول الملح الفلزي المذاب بالماء مع محاليل الليكندات BH, TH, L الدذابة بالماء والايثانول بنفس النسب المستخدمة في اعلاه، ثم اضيف إلى المزيج محلول هيدروكسيد البوتاسيوم (2M)(المذاب بالماء بالتدريج قطرة فقطرة لحين اكتمال الترسيب، رشح الراسب وغسل بالماء المقطر ثم بثائي اثيل وجفف ووزن والجدول يبين الخصائص الفيزيائية ونسب الاوزان لليكندات في تحضيرالمعقدات كما في المعادلتين : $\mathrm{Co}\left(\mathrm{CH}_{3} \mathrm{COO}\right)_{2} .4 \mathrm{H}_{2} \mathrm{O}+\mathrm{L}_{1} \mathrm{H}+\mathrm{TH}+\mathrm{GH}+\mathrm{KOH} \rightarrow\left[\mathrm{Co}\left(\mathrm{L}_{1}\right)(\mathrm{G})(\mathrm{TH})\right]_{2}\left(\mathrm{CH}_{3} \mathrm{COOK}\right)_{2}+6 \mathrm{H}_{2} \mathrm{O} \ldots .$. (1) $\mathrm{Co}\left(\mathrm{CH}_{3} \mathrm{COO}\right)_{2} .4 \mathrm{H}_{2} \mathrm{O}+\mathrm{L}_{1} \mathrm{H}+\mathrm{TH}+\mathrm{mf}+\mathrm{KOH} \rightarrow\left[\mathrm{Co}\left(\mathrm{L}_{1}\right)(m f)(\mathrm{T})\right]_{2}\left(\mathrm{CH}_{3} \mathrm{COOK}\right)_{2}+6 \mathrm{H}_{2} \mathrm{O} \ldots \ldots$ (2)

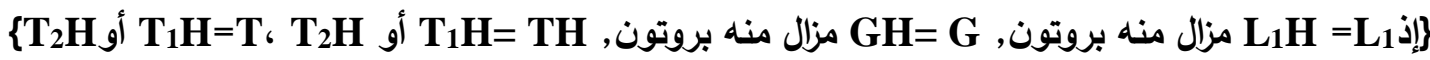


الجدول (2) الاوزان والنسب وظروف التفاعل المتوقعة للمعقدات المحضرة

\begin{tabular}{|c|c|c|c|c|c|c|c|c|c|c|}
\hline ت & صيغة الملح & $\begin{array}{l}\text { وزن } \\
\mathbf{T}_{1} \mathbf{H}\end{array}$ & $\begin{array}{l}\text { وزن } \\
\mathbf{T}_{2} \mathbf{H}\end{array}$ & وزن & وزن & $\begin{array}{l}\text { وزن } \\
\text { Mf }\end{array}$ & وزن & $\begin{array}{l}\text { الحامضبة } \\
\text { PH }\end{array}$ & الناتج & الصيغة المتوقعة للمُعقّدات \\
\hline 1. & $\mathrm{Co}\left(\mathrm{CH}_{3} \mathrm{CO}_{2}\right)_{2} \cdot 4 \mathrm{H}_{2} \mathrm{O}$ & 0.40 & - & 0.24 & - & - & 0.14 & $6.5-7$ & $93 \%$ & {$\left[\mathrm{Co}\left(\mathrm{T}_{1} \mathrm{H}\right)\left(\mathrm{L}_{1} \mathrm{H}\right)(\mathrm{GH})\right]\left(\mathrm{CH}_{3} \mathrm{CO}_{2}\right)_{2}$} \\
\hline 2. & $\mathrm{Co}\left(\mathrm{CH}_{3} \mathrm{CO}_{2}\right)_{2} \cdot 4 \mathrm{H}_{2} \mathrm{O}$ & 0.40 & - & 0.24 & - & - & 0.14 & $9.5-10$ & $78 \%$ & {$\left[\mathrm{Co}\left(\mathrm{T}_{1} \mathrm{H}\right)\left(\mathrm{L}_{1}\right)(\mathrm{G})\right]$} \\
\hline 3. & $\mathrm{Co}\left(\mathrm{CH}_{3} \mathrm{CO}_{2}\right)_{2} \cdot 4 \mathrm{H}_{2} \mathrm{O}$ & - & 0.35 & 0.24 & - & - & 0.14 & $6.5-7$ & $85 \%$ & {$\left[\mathrm{Co}\left(\mathrm{T}_{2} \mathrm{H}\right)\left(\mathrm{L}_{1} \mathrm{H}\right)(\mathrm{GH})\right]\left(\mathrm{CH}_{3} \mathrm{CO}_{2}\right)_{2}$} \\
\hline 4. & $\mathrm{Co}\left(\mathrm{CH}_{3} \mathrm{CO}_{2}\right)_{2} \cdot 4 \mathrm{H}_{2} \mathrm{O}$ & - & 0.35 & 0.24 & - & - & 0.14 & $9.5-10$ & $75 \%$ & {$\left[\mathrm{Co}\left(\mathrm{T}_{2} \mathrm{H}\right)\left(\mathrm{L}_{1}\right)(\mathrm{G})\right]$} \\
\hline 5. & $\mathrm{Co}\left(\mathrm{CH}_{3} \mathrm{CO}_{2}\right)_{2} \cdot 4 \mathrm{H}_{2} \mathrm{O}$ & 0.40 & - & 0.24 & - & 0.25 & - & $6.5-7$ & $93 \%$ & {$\left[\mathrm{Co}\left(\mathrm{T}_{1} \mathrm{H}\right)\left(\mathrm{L}_{1} \mathrm{H}\right)(\mathrm{mf})\right]\left(\mathrm{CH}_{3} \mathrm{CO}_{2}\right)_{2}$} \\
\hline 6. & $\mathrm{Co}\left(\mathrm{CH}_{3} \mathrm{CO}_{2}\right)_{2} \cdot 4 \mathrm{H}_{2} \mathrm{O}$ & 0.40 & - & 0.24 & - & 0.25 & - & $9.5-10$ & $86 \%$ & {$\left[\mathrm{Co}\left(\mathrm{T}_{1}\right)\left(\mathrm{L}_{1}\right)(\mathrm{mf})\right]$} \\
\hline 7. & $\mathrm{Co}\left(\mathrm{CH}_{3} \mathrm{CO}_{2}\right)_{2} \cdot 4 \mathrm{H}_{2} \mathrm{O}$ & - & 0.35 & 0.24 & - & 0.25 & - & $6.5-7$ & $84 \%$ & {$\left[\mathrm{Co}\left(\mathrm{T}_{2} \mathrm{H}\right)\left(\mathrm{L}_{1} \mathrm{H}\right)(\mathrm{mf})\right]\left(\mathrm{CH}_{3} \mathrm{CO}_{2}\right)_{2}$} \\
\hline 8. & $\mathrm{Co}\left(\mathrm{CH}_{3} \mathrm{CO}_{2}\right)_{2} \cdot 4 \mathrm{H}_{2} \mathrm{O}$ & - & 0.35 & 0.24 & - & 0.25 & - & $9.5-10$ & $77 \%$ & {$\left[\mathrm{Co}\left(\mathrm{T}_{1}\right)\left(\mathrm{L}_{1}\right)(\mathrm{mf})\right]$} \\
\hline
\end{tabular}

4- التقانات المستخدمة للتشخيص:

1.4- القياسات التحليلية:

قدرت كمية الكوبلت (Co) (II) في المعقدات باستخدام جهاز طيف الامتصاص الذري (Atomic absorption) نوع Sens AAGS Scintific Equipment قسم التحليلات المرضية في الموصل- العراق. وقيس التحليل الدقيق للعناصر (CHNS) للمركبات (22) في قسم الكيمياء كلية العلوم- جامعة طهران - ايران بجهاز من نوع (Elementarel III). 2.4-القياسات الفيزيائية:

قيست درجات الانصهار لجميع المركبات بجهاز الانصهار الكهروحراري (9300) وبجهاز جهاز Electro Thermal TA

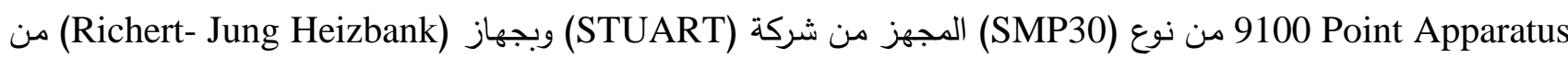
نوع (WME) وقد تم القياس في مختبر الكيمياء العضوية - قسم الكيمياء-كلية التربية للعلوم الصرفة - جامعة الموصل ـ وقيست التوصيلية الكهربائية المولاريـة بجهاز التوصيل الكهربائي من نوع (Wtarter 3100c) وباستعمال المذيب فُشائي مثيل سلفوكسايد وبتركيز (DMSO) (25²C) في مختبرات قسم الكيمياء- كلية التربية للعلوم الصرفة- جامعة تكريتمحافظة صلاح الدين- العراق وكما قيس الوزن الجزيئي النسبي لجميع المركبات قيد الدرس (23) باستعمال طريقة الإنخفاض بدرجة الانجماد (الكرايوسكوب-Cryoscopically)كما موضـح في الثكل (1). قيس الحث الكهرومغناطيسي باستعمال جهاز 455 DSP Gaussmeter) للمعقدات قيد الدرس باستعمال جهاز من نوع (Magnetic Susceptibility Balance) المستورد من شركة ( Sherwood (Cambridge) منشأها (Caientific MK1 الدين - العراق. 
Spectrometer (V-530) Jasco UV-Vis قيست الأطياف الالكترونية للمركبات قيد الدرس باستعمال جهاز

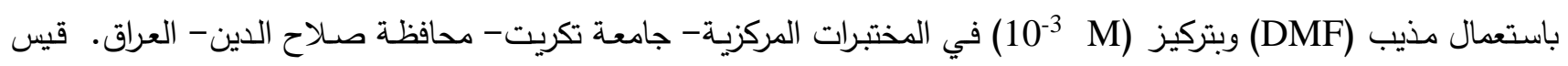

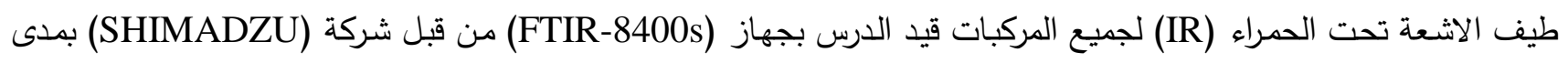
(400-4000 cm²-1), وذلك بسحق العينة وكبسها على شكل اقراص مع KBr تم القياس في المختبرات المركزية- جامعة تكريتمحافظة صلاح الدين - العراق. شععت جميع المركبات قيد الدرس باستعمال جهاز ليزري (Laser) نوع هيليون- نيون (He-Ne)

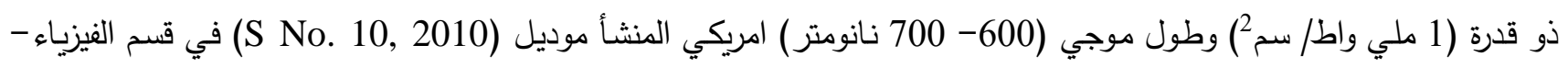

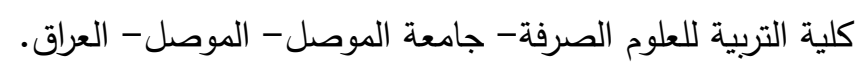
6- تقييم الفعالية المضادة للبكتريا:

اختبرت الفعّاليّة البكتيرية لجميع المركبات قيد الدراسة على البكتريا المرضية Escherichia.coli وتم تسجيل النسبة

المئوية للتثبيط.(24).و Staphylococcus aureus و Klipsila pnnemoia و و Pاستعمال تقانة الانتشار على سطح الاكار، عزلت البكتريا وشخصت باستخدام طريقة قياسية (26,25) قبل استخدامها، تم قياس منطقة التثبيط (27)، اخذت تراكيز مختلفة من المواد قيد الدراسة (500، 250، 125، 62.5 مايكروغرام/مل) لحساب أقل تركيز مثبط (MIC) وقورنت مع مضاد حيوي وهو السبرومايسين.

\section{النتائج والمناقشة}

أوضحت نتائج القياسات التحليلية ان نسبة العناصر (الكوبلت والكاربون والهيدروجين والنتروجين والكبريت) كانت متفقة مع صيخ المعقدات المقترحة كما موضح في الجدول أدناه:

الجدول (3) النسبة المئوية لعناصر المققدات المحضرة

\begin{tabular}{|c|c|c|c|c|c|}
\hline$ت$ & \%Co (عمليًا)(نظرياً & \% (عليا)نظريا & \% \% (عليا)نظريا & N\%(عليا)نظريا & \%(عليا):نظريا \\
\hline 1. & $9.92(9.90)$ & $38.42(36.41)$ & $4.92(4.91)$ & $16.51(16.50)$ & $5.40(5.40)$ \\
\hline 2. & $12.39(12.35)$ & $37.86(37.85)$ & $4.87(4.85)$ & $20.61(20.61)$ & $6.74(6.73)$ \\
\hline 3. & $9.51(9.51)$ & 40.71 (40.71) & $5.53(5.53)$ & $15.83(15.83)$ & $5.17(5.17)$ \\
\hline 4. & 11.75 (11.75) & $40.72(40.75)$ & $5.63(5.63)$ & 19.55 (19.55) & 6.39 (6.39) \\
\hline 5. & $9.09(9.06)$ & $38.92(38.90)$ & $5.44(5.40)$ & $23.78(23.75)$ & $4.95(4.92)$ \\
\hline 6. & 11.48 (11.48) & 39.65 (39.66) & $5.48(5.48)$ & $27.20(27.20)$ & $6.88(6.86)$ \\
\hline 7. & $8.57(8.60)$ & 41.92 (91.90) & $6.16(6.14)$ & $22.41(22.40)$ & $4.66(4.66)$ \\
\hline 8. & $10.61(10.66)$ & $41.08(41.05)$ & $6.17(6.15)$ & $27.73(27.75)$ & $5.77(5.75)$ \\
\hline
\end{tabular}

أثبتت النتائج اعلاه انها متفقة تماماً مع النسب المولية للتفاعل 1:1:1:1 الملح الفلزي: BH:TH:L1H، ان المعقدات مواد

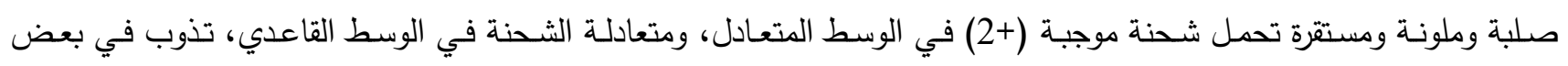
المذيبات العضوية (DMSO, DMF) وشحيحة الذوبان جداً في ثنائي أثيل ايثر وايثر بترولي، وتتصهر جميع المعقدات ، و وكما

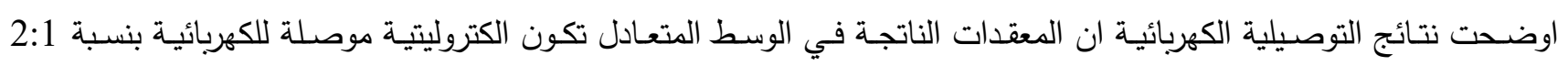

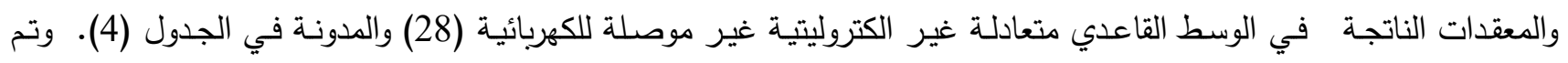
حساب الوزن الجزيئي النسبي لجميع المركبات قيد الدراسة من العلاقة الخطية التي تم الحصول عليها من الرسم (شكل 1) والقيم 
المدونـة في الجدول (4). وبينت نتائج الحث الكهرومغناطيسي أنَّ بعض المعقدات قيد الدراسـة تمتلك قوة كهرومغناطيسية والقيم

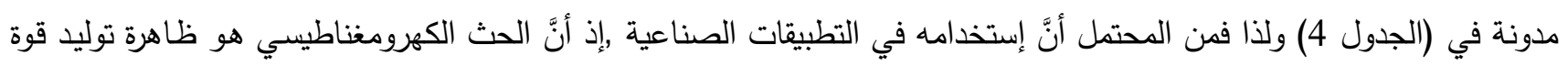
دافعة كهربائية تأثيرية وتيار مؤثر في موصل بتأثير المجال المغناطيسي وتعد الظاهرة مهمة جداً إذ لها تطبيقات عديدة (30,29),

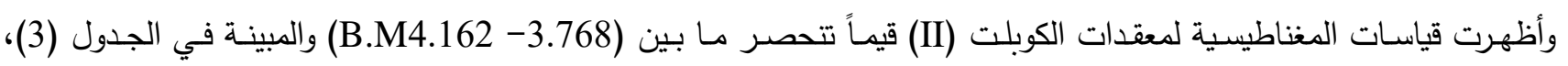

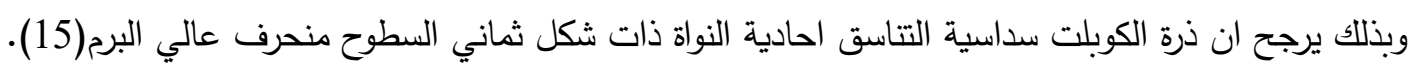

الجدول (4) الخواص الفيزيائية للمعقدات المحضرة

\begin{tabular}{|c|c|c|c|c|c|c|c|}
\hline الوزن الجزيئي & $\begin{array}{c}\text { العزم المغناطيسي الفعال } \\
\text { (B.M.) Meff }\end{array}$ & الانجماد (مُجمات & $\begin{array}{c}\text { التوصيليّة المولارية } \\
\text { 1lohms } \\
\end{array}$ & الحث & 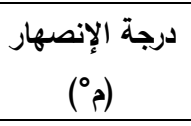 & اللون & ت \\
\hline $593.9(593.11)$ & 3.875 & -22.5 & 133 & 79 & 200 & ارجواني & .1 \\
\hline $475.8(475.5)$ & 3.887 & -18.5 & 38 & 35 & 220 & ارجواني & .2 \\
\hline 619.5 (619.6) & 4.070 & -25.5 & 141 & 54 & 245 & عنابي داكن & .3 \\
\hline $501.4(501.4)$ & 4.065 & -17.5 & 36 & 78 & 240 & عنابي & .4 \\
\hline $648.0(648.5)$ & 4.011 & -21.5 & 138 & 39 & 202 & بني فاتح & .5 \\
\hline $514.9(514.8)$ & 3.780 & -17.5 & 25 & 41 & 210 & بني غامق & .6 \\
\hline $687.7(687.9)$ & 3.965 & -27.5 & 110 & 39 & 193 & زيتوني داكن & .7 \\
\hline $555.5(555.7)$ & 3.786 & -17.5 & 27 & 41 & 243 & زيتوني فاتح & .8 \\
\hline
\end{tabular}

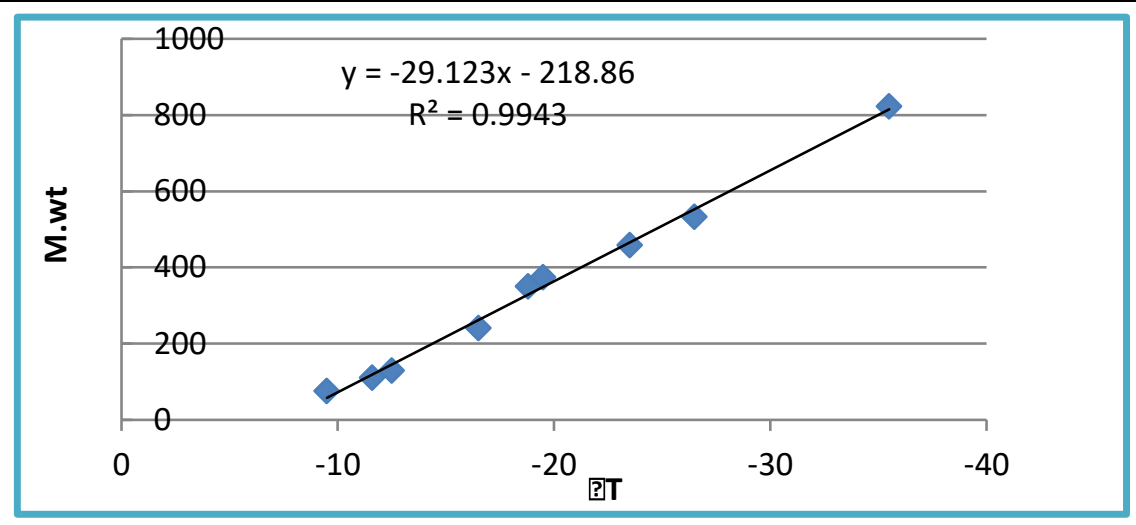

\section{شكل (1) تقدير الوزن الجزيبي النسبي}

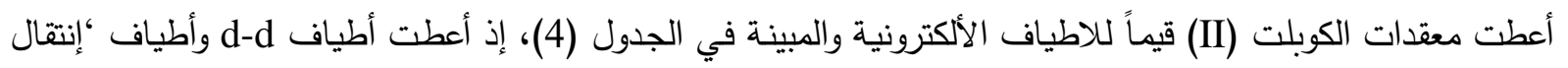

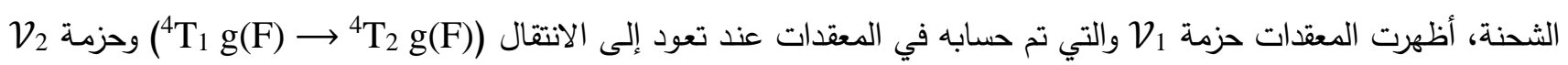
عند تعود إلى الانتقال (

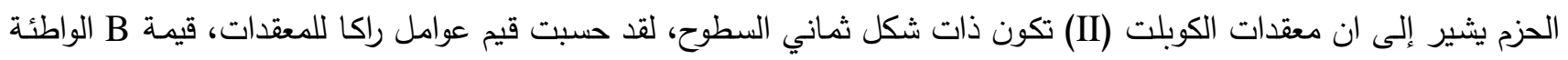

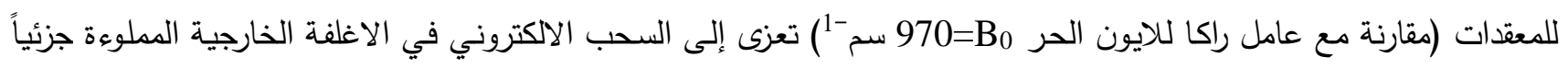
للكوبلت (II) بتأثير المجال، فكلما قلت قيمة B ازداد السحب فتزداد بذللك الخاصية التساهية للاصرة بين الفلز والليكند، وتبين قيمة

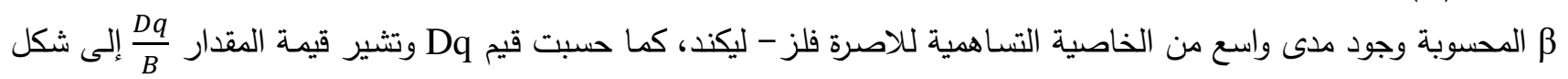
المعقد, والجدول (4) يوضح جميع حزم الانتقال الالكتروني وقيم عوامل راكا (31) والاشكال (3,2) ايضاً .

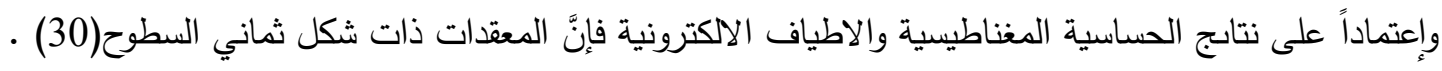


الجدول(5) الاطياف الاككترونية وعوامل راكا

\begin{tabular}{|c|c|c|c|c|c|c|c|c|c|c|}
\hline $\boldsymbol{\nu}_{3} / \boldsymbol{\nu}_{2}$ & $\nu_{3} / \nu_{1}$ & $\boldsymbol{\nu}_{2} / \nu_{1}$ & $\boldsymbol{D}_{q} / \boldsymbol{\beta}$ & $\begin{array}{c}10 D_{q} \\
\left(\mathrm{~cm}^{-1}\right)\end{array}$ & $\begin{array}{c}B \\
\left(\mathrm{~cm}^{-1}\right)\end{array}$ & $\begin{array}{c}C . T \\
\left(\mathrm{~cm}^{-1}\right)\end{array}$ & $\begin{array}{c}\mathcal{\nu}_{3} \\
\left(\mathrm{~cm}^{-1}\right)\end{array}$ & $\begin{array}{c}\boldsymbol{\nu}_{2} \\
\left(\mathrm{~cm}^{-1}\right)\end{array}$ & $\begin{array}{c}\boldsymbol{\nu}_{\boldsymbol{1}} \\
\left(\mathrm{cm}^{-1}\right)\end{array}$ & 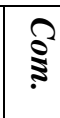 \\
\hline 1.28 & 2.768 & 2.151 & 0.8 & 7094 & 885.6 & 27173.9 & 19551 & 15015 & 7063 & 1. \\
\hline 1.24 & 2.665 & 2.148 & 0.82 & 7000 & 849.2 & 27173.9 & 18726 & 15015 & 7296 & 2. \\
\hline 1.129 & 2.414 & 2.139 & 0.97 & 7027 & 729.0 & 27173.9 & 17001 & 15015 & 7344 & 3. \\
\hline 1.19 & 2.572 & 2.145 & 0.87 & 7433 & 852.5 & 27173.9 & 19157 & 15015 & 7448 & 4. \\
\hline 1.19 & 2.572 & 2.145 & 0.87 & 7433 & 852.5 & 27173.9 & 19157 & 15015 & 7448 & 5. \\
\hline 1.07 & 2.283 & 2.133 & 1.02 & 7348 & 715.2 & 34.013 .6 & 17301 & 15873 & 7578 & 6. \\
\hline 1.12 & 2.414 & 2.139 & 0.96 & 7414 & 765.7 & 42.016 .8 & 17857 & 15873 & 7397 & 7. \\
\hline 1.24 & 2.665 & 2.148 & 0.82 & 7001 & 849.2 & 27173.9 & 18726 & 15015 & 7026 & 8. \\
\hline
\end{tabular}




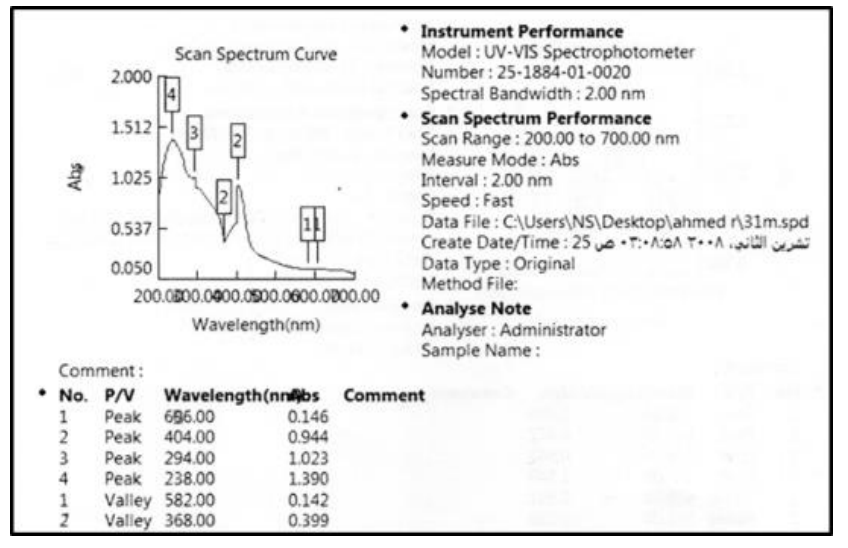

الشكل (3) الاطياف الالكترونية للمعقد (8)

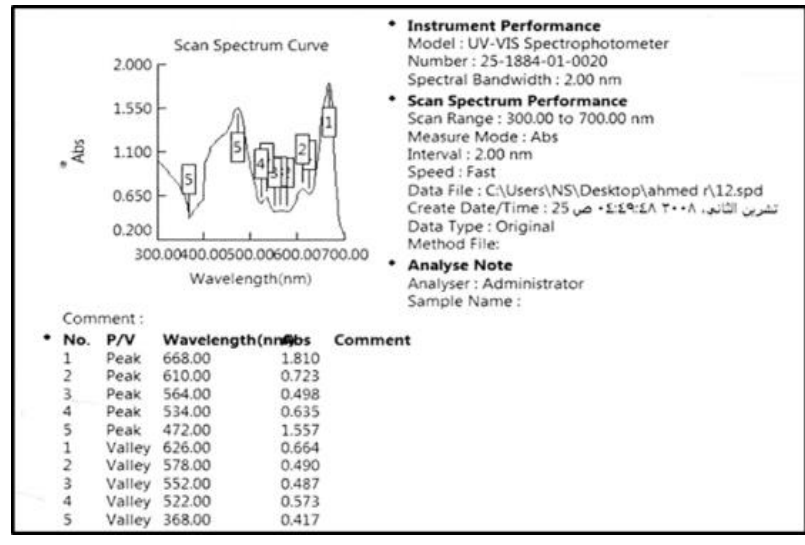

الثكل (2) الاطياف الاككترونية للمققد (5)

سجلت أطياف الأشعة تحت الحمراء لليكندات والمعقدات قيد الدراسـة في المدى(400-000 سم-1) والمدونة في

الجدول (5) والاشكال (5,4) توضح اطياف الاشعة تحت الحمراء لليكندات والمعقدات المحضرة.

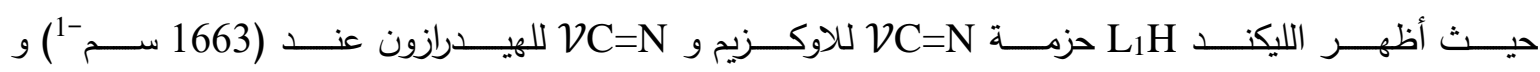
(1643 سم-1') على التوالي، ولوحظ في طيف المعقدات انهما ازيحتا نحو تردد أوطأ في جميع المعقدات وتحت جميع الظروف التحضيرية (1592-1600 سم -1) دلالة على تتاسق ذرتي النتروجين من مجموعتي الازوميثين مع الأيون الفلزي (33,32)، وتشير

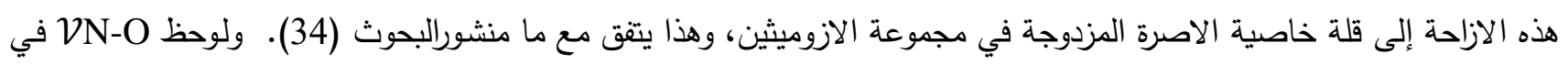

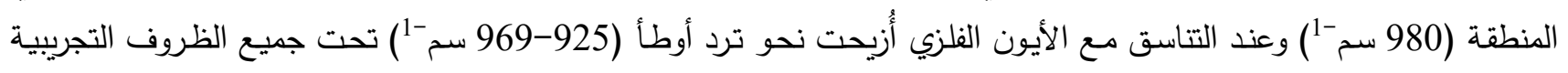

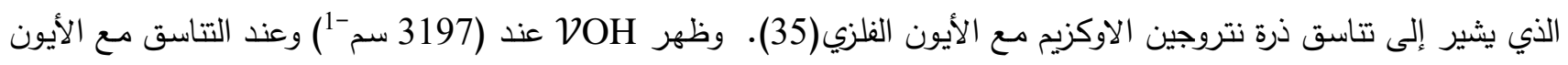

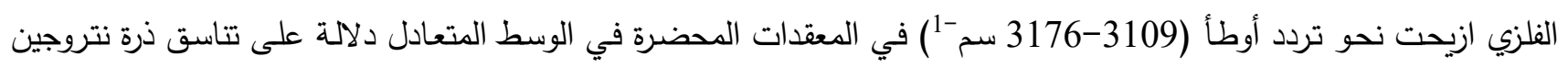

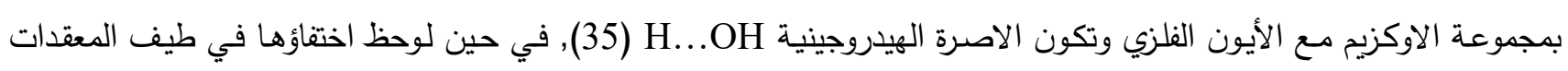

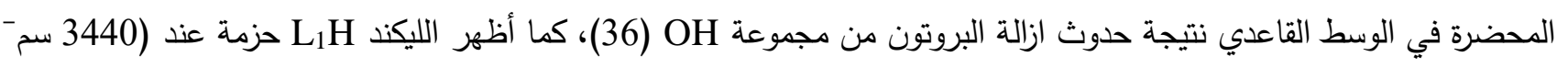
1 ${ }^{1}$ في طيف الليكندين TC=N ازيحت نحو تردد أوطأ (1630-1654 سم -1) في طيف جميع المعقدات وتحت جميع الظروف التجريبية، تشير إلى حدوث التتاسق مع الأيون الفلزي وتؤكد قلة خاصية الاصرة المزدوجة في مجموعة الازوميثين، وهذا متفق مع ما نشور من بحوث (34). وأعطى

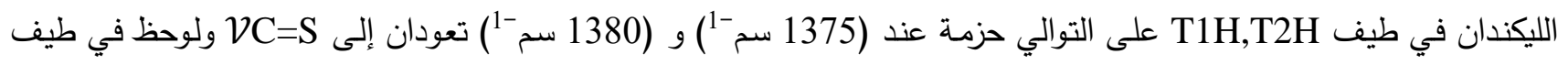

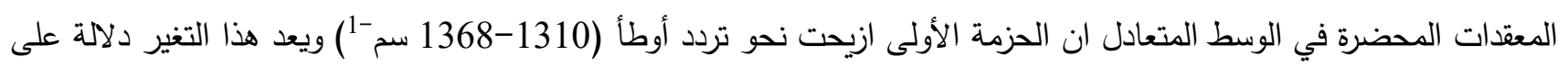
تتاسق ذرة كبريت مجموعة الثايون مع الأيون الفلزي (38) • وقد لوحظ اختفاء هذه الحزمة في طيف المعقدات المحضرة في الوسط

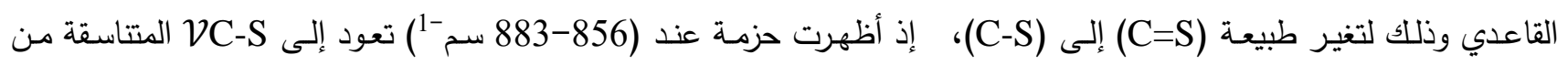
خلال ذرة الكبريت مع الأيون الفلزي (40,39)، كما أظهر طيف ليكندي T2H, T على التوالي تعود إلى VNH، وعند التناسق يصبح الحال اكثر صعوبة وذلك لوجود عوامل عديدة كتأثير التتاسق (يؤدي إلى ازاحة الحزمة نحو تردد أوطأ وتأثير الاصرة الهيدروجينية عند التناسق (يؤدي إلى حدوث الىاحة نحو تردد أعلى) فضلاً عن وجود مجاميع

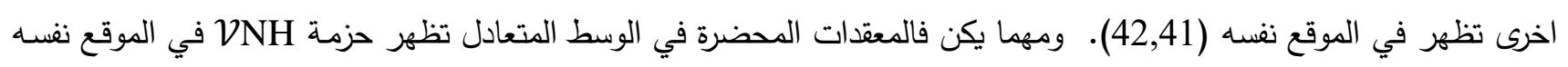
تقريباً، ومن المؤكد عدم وجود تتاسق بين هذه المجموعة والايون الفلزي، ولكن في حالة المعقدات المحضرة في الوسط القاعدي 
يتعذر ملاحظة اختفاؤها بسبب وجود الاواصر الهيدروجينية، وعلى اية حال فمن المؤكد اختفاء هذه المحزمة بسبب الثكل الثايولي

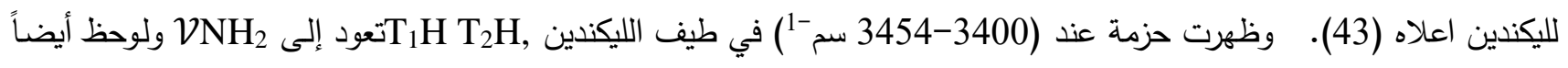

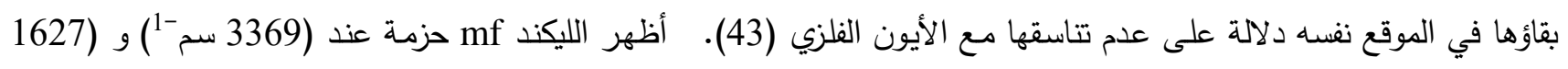

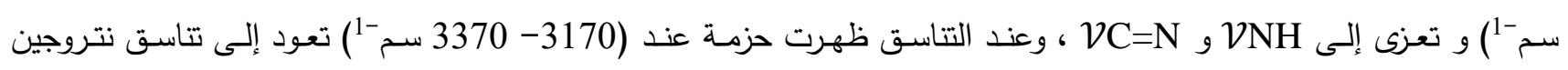

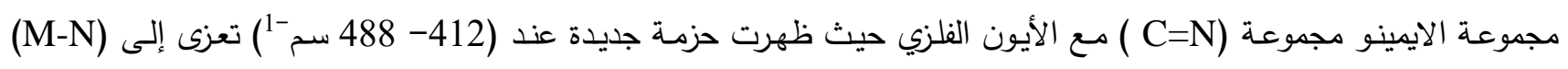

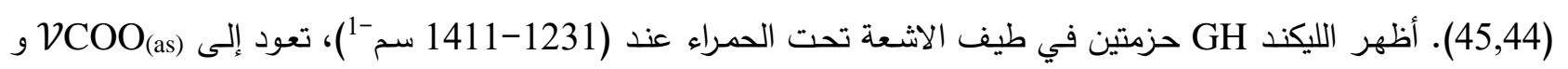

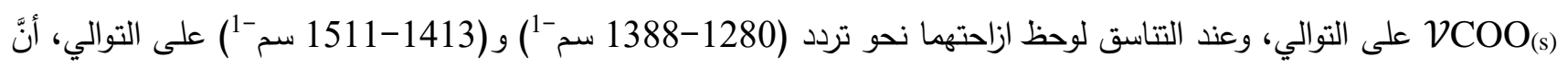
الفرق بين تناسق مجموعة الكاروكسيل، ولقد لوحظت القيم (1413-1511 سم+1) دلالة على أن مجموعة الكاربوكسيل قد تتاسقت مع الأيون

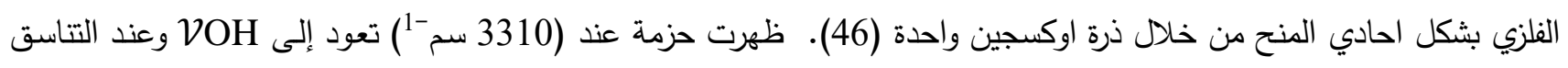
لوحظ ازاحتها نحو تردد أوطأ في طيف المعقدات المحضرة في الوسط المتعادل قليلاً مما يدل على تناسق مجموعة خلال ذرة الاوكسجين، في حين لوحظ اختفاؤها نتيجة ازالة البروتون منها في طيف المعقدات المحضرة في الوسط القاعدي (46).

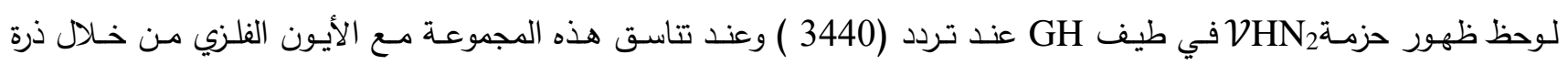
النتروجين أُزيحت نحو تردد واطئ (3350-3390سم-1 ) (47). أظهر طيف المعقدات المحضرة في الوسط المتعادل تقريباً عند

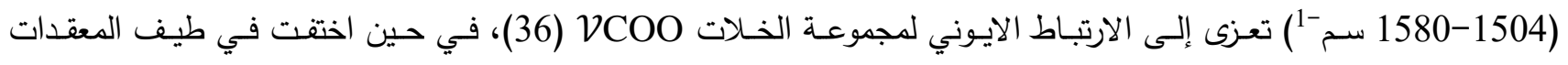

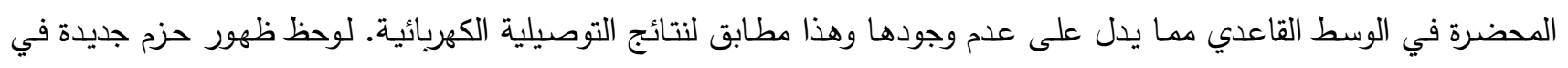

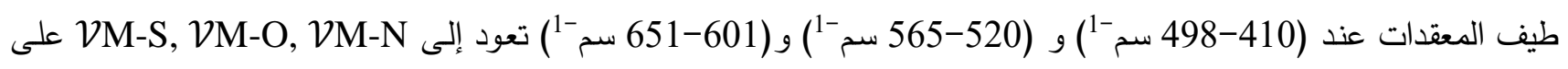
التوالي، أنَّ ظهور هذه الحزم يعزز تكوّن المُعقّدات (38). 
Journal of Education and Science (ISSN 1812-125X), Vol: 30, No: 2, 2021 (1-18)

الجدول(6) طيف الاشعة تحت الحمراء لليكندات وامعقدات المحضرة

\begin{tabular}{|c|c|c|c|c|c|c|c|c|c|c|c|c|c|c|c|c|c|c|}
\hline \multicolumn{3}{|c|}{ حزم جليدة } & \multirow{2}{*}{$\begin{array}{c}A c . \\
\mathrm{CO}_{2} \boldsymbol{a s} \\
\mathrm{CO}_{2} \mathrm{~S}\end{array}$} & \multicolumn{2}{|c|}{$M f$} & \multicolumn{4}{|c|}{$G H$} & \multicolumn{3}{|c|}{$T 1 H \& T 2 H$} & \multicolumn{5}{|c|}{$L 1 H \& L 2 H$} & \multirow[b]{2}{*}{ No } \\
\hline$M-S$ & $M-O$ & $M-N$ & & $N-H$ & $C=N$ & $\mathrm{NH}_{2}$ & $\Delta \boldsymbol{\nu}$ & $\mathrm{CO}_{2} a s$ & $\mathrm{CO}_{2} \mathrm{~S}$ & $N H$ & $C=N$ & $\begin{array}{c}C=S \\
C-S\end{array}$ & $\mathrm{NH}_{2}$ & $\begin{array}{c}\mathrm{OH} \\
\mathrm{N}-\mathrm{O}\end{array}$ & $C=N_{H}$ & $C-N_{o}$ & $C=N_{\text {sch }}$ & \\
\hline- & - & - & - & - & - & - & - & - & - & - & - & - & 3440 & $\begin{array}{c}3197 \\
980\end{array}$ & 1643 & 1680 & 1663 & $\mathrm{~L}_{1} \mathrm{H}$ \\
\hline- & - & - & - & - & - & - & - & - & - & - & - & - & 3454 & $\begin{array}{c}3198 \\
999\end{array}$ & 1638 & 1683 & 1675 & $\mathrm{~L}_{2} \mathrm{H}$ \\
\hline- & - & - & - & - & - & - & - & - & - & 3247 & 1647 & 1375 & - & - & - & - & - & $\mathrm{T}_{1} \mathrm{H}$ \\
\hline- & - & - & - & - & - & 3415 & 180 & 1411 & 1231 & - & - & - & - & - & - & - & - & $\mathrm{GH}$ \\
\hline- & - & - & - & 3369 & 1627 & - & - & - & - & - & - & - & - & - & - & - & - & Mf \\
\hline 611 & 547 & $\begin{array}{l}410-436 \\
451-495 \\
\end{array}$ & $\begin{array}{l}1517 \\
1548 \\
\end{array}$ & - & - & 3391 & 148 & 1448 & 1300 & 3261 & 1630 & $\begin{array}{c}1320 \\
883 \\
\end{array}$ & 3395 & $\begin{array}{c}3164 \\
925 \\
\end{array}$ & 1598 & 1600 & 1627 & .1 \\
\hline 651 & 518 & $\begin{array}{l}415-432 \\
457-486\end{array}$ & & - & - & 3320 & 108 & 1413 & 1305 & & 1645 & 869 & 3407 & $\begin{array}{c}3153 \\
927\end{array}$ & 1594 & 1635 & 1611 & .2 \\
\hline 617 & 532 & $\begin{array}{l}423-455 \\
367-492\end{array}$ & $\begin{array}{l}1417 \\
1588\end{array}$ & - & - & 3300 & 114 & 1454 & 1340 & 3292 & 1645 & $\begin{array}{c}1319 \\
881\end{array}$ & 3420 & $\begin{array}{c}3160 \\
939\end{array}$ & 1596 & 1620 & 1600 & .3 \\
\hline 617 & 543 & $\begin{array}{l}412-432 \\
468-412 \\
\end{array}$ & & - & - & 3363 & 117 & 1417 & 1300 & - & 1645 & 881 & 3400 & $\begin{array}{c}3175 \\
969 \\
\end{array}$ & 1596 & 1612 & 1636 & .4 \\
\hline 611 & 557 & $\begin{array}{l}412-435 \\
451-487\end{array}$ & $\begin{array}{l}1504 \\
1584\end{array}$ & 3321 & 1568 & - & - & - & - & 3303 & 1645 & $\begin{array}{c}1344 \\
869\end{array}$ & 3435 & $\begin{array}{c}3155 \\
948\end{array}$ & 1592 & 1623 & 1612 & .5 \\
\hline 627 & 534 & $\begin{array}{l}431-445 \\
476-498 \\
\end{array}$ & & 3310 & 1587 & - & - & - & - & & 1647 & 867 & 3355 & $\begin{array}{c}3143 \\
946 \\
\end{array}$ & 1597 & 1623 & 1610 & .6 \\
\hline 613 & 565 & $\begin{array}{l}413-435 \\
464-498 \\
\end{array}$ & & 3350 & 1566 & - & - & - & - & 3255 & 1644 & 869 & 3334 & $\begin{array}{c}3153 \\
948 \\
\end{array}$ & 1597 & 1624 & 1600 & .7 \\
\hline 638 & 520 & $\begin{array}{l}408-423 \\
449-495\end{array}$ & & $3340-$ & 1546 & 3344 & 159 & 1467 & 1308 & & 1645 & 869 & 3415 & $\begin{array}{c}3153 \\
931 \\
\end{array}$ & 1598 & 1652 & 1608 & .8 \\
\hline
\end{tabular}


Journal of Education and Science (ISSN 1812-125X), Vol: 30, No: 2, $2021(1-18)$

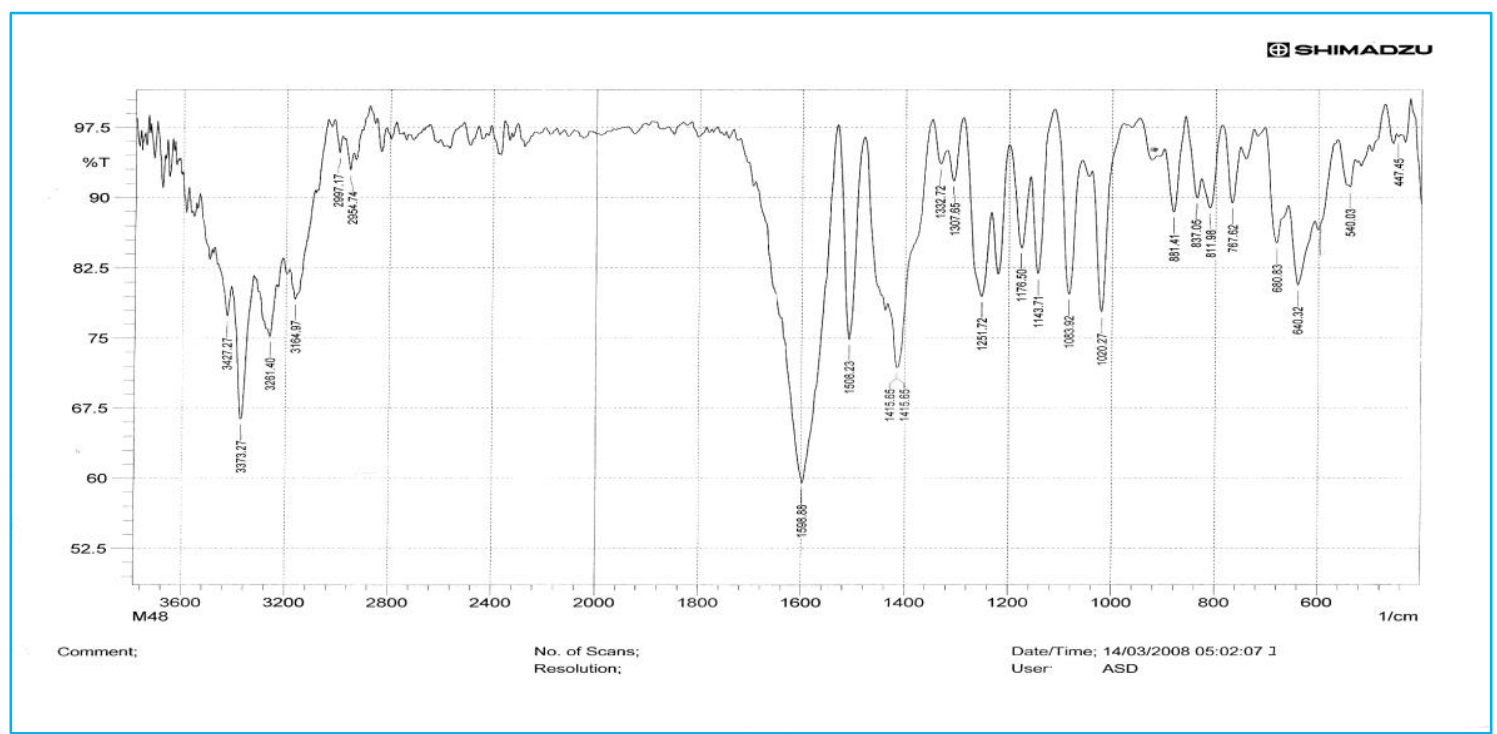

الثكل (9) طيف الألمعقد (5)

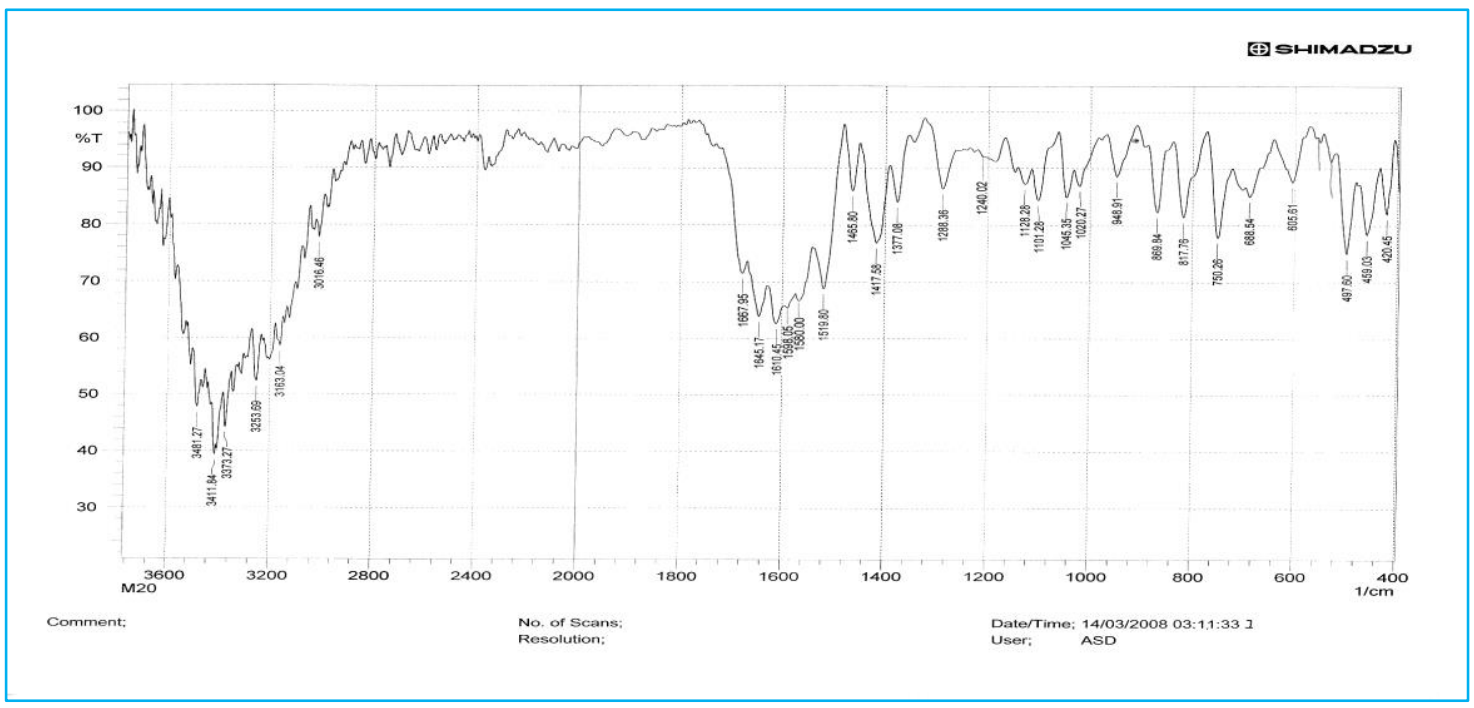

الثكل(10) طيف ال ال المعقد (8)

تم دراسة تأثير اشعة الليزر (Ne-He) ذا الطول الموجي (600- 700 نانومتر) لليكندات والمعقدات ولوحظ عدم الأميات

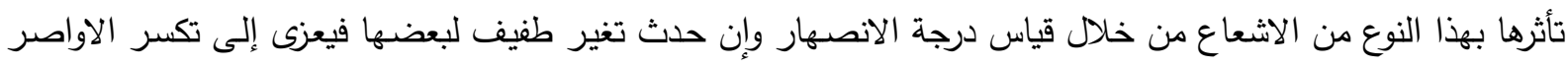
الهيدروينية (52)، كما مبين في الجدولين (9,8,7) ادناه. 
الجدول (7) نتائج تشعيع الليكندات باشعة الليزر

\begin{tabular}{|c|c|c|c|c|c|c|c|c|c|c|c|c|c|c|}
\hline نُخ & $\stackrel{\mathscr{\Xi}}{\Xi}$ & ه & بَّ & $\underset{\Xi}{\Xi}$ & 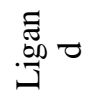 & $\stackrel{\text { : }}{\Sigma}$ & $\stackrel{\mathscr{g}}{\Xi}$ & סี & 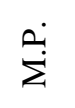 & $\stackrel{\mathscr{\Xi}}{\Xi}$ & סี & نَّ & $\underset{\Xi}{\Xi}$ & סี \\
\hline 198 & 0.0 & \multirow{5}{*}{$\underset{E}{\stackrel{\Xi}{\Xi}}$} & 201 & 0.0 & \multirow{5}{*}{$\underset{\sim}{\stackrel{I}{N}}$} & 348 & 0.0 & \multirow{5}{*}{$\stackrel{\Xi}{\Xi}$} & 226 & 0.0 & \multirow{5}{*}{$\overleftrightarrow{\Xi}$} & 280 & 0.0 & \multirow{5}{*}{$\stackrel{\mathbb{J}}{J}$} \\
\hline 198 & 0.5 & & 201 & 0.5 & & 348 & 0.5 & & 226 & 0.5 & & 280 & 0.5 & \\
\hline 198 & 1.0 & & 201 & 1.0 & & 348 & 1.0 & & 226 & 1.0 & & 280 & 1.0 & \\
\hline 198 & 1.5 & & 201 & 1.5 & & 348 & 1.5 & & 226 & 1.5 & & 280 & 1.5 & \\
\hline 198 & 2.0 & & 201 & 2.0 & & 348 & 2.0 & & 226 & 2.0 & & 280 & 2.0 & \\
\hline
\end{tabular}

الجدول(8) نتائج تشعيع المُعقّدات المحضرة في الوسط المتعادل بأشعة الليزر

\begin{tabular}{|c|c|c|c|c|c|c|c|c|c|c|c|}
\hline$\stackrel{\dot{\square}}{\dot{\Sigma}}$ & $\stackrel{\mathscr{\Xi}}{\Xi}$ & 言 & $\stackrel{\dot{\leftrightarrow}}{\dot{\Sigma}}$ & $\stackrel{\mathscr{I}}{\Xi}$ & 远 & $\stackrel{\dot{\Sigma}}{\dot{\Sigma}}$ & $\stackrel{\mathscr{\Xi}}{\Xi}$ & 己气 & $\stackrel{\circ}{\Sigma}$ & $\stackrel{\mathscr{\Xi}}{\Xi}$ & 葛 \\
\hline 240 & 0.0 & \multirow{5}{*}{7} & 245 & 0.0 & \multirow{5}{*}{5} & 220 & 0.0 & \multirow{5}{*}{3} & 200 & 0.0 & \multirow{5}{*}{1} \\
\hline 240 & 0.5 & & 245 & 0.5 & & 220 & 0.5 & & 200 & 0.5 & \\
\hline 240 & 1.0 & & $240^{*}$ & 1.0 & & 220 & 1.0 & & 200 & 1.0 & \\
\hline 240 & 1.5 & & $240^{*}$ & 1.5 & & 220 & 1.5 & & 200 & 1.5 & \\
\hline 240 & 2.0 & & $240^{*}$ & 2.0 & & 220 & 2.0 & & 200 & 2.0 & \\
\hline
\end{tabular}

الجدول(9) نتائج تشعيع المعقدات المحضرة في الوسط القاعدي بأشعة الليزر

\begin{tabular}{|c|c|c|c|c|c|c|c|c|c|c|c|}
\hline$\stackrel{\dot{\Sigma}}{\dot{\Sigma}}$ & $\stackrel{\mathscr{B}}{\Xi}$ & 言 & $\stackrel{\dot{\Sigma}}{\dot{\Sigma}}$ & $\underset{\Xi}{\stackrel{\Xi}{\Xi}}$ & 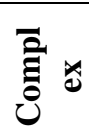 & 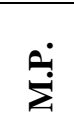 & $\stackrel{\Xi}{\Xi}$ & 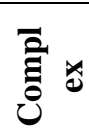 & 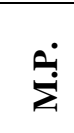 & $\stackrel{\mathscr{\Xi}}{\stackrel{\Xi}{\mid}}$ & 产 \\
\hline 240 & 0.0 & \multirow{5}{*}{8} & 275 & 0.0 & \multirow{5}{*}{6} & 220 & 0.0 & \multirow{5}{*}{4} & 200 & 0.0 & \multirow{5}{*}{2} \\
\hline 240 & 0.5 & & 275 & 0.5 & & 220 & 0.5 & & 190 & 0.5 & \\
\hline $245^{*}$ & 1.0 & & 275 & 1.0 & & 220 & 1.0 & & 190 & 1.0 & \\
\hline $245^{*}$ & 1.5 & & 275 & 1.5 & & 220 & 1.5 & & 190 & 1.5 & \\
\hline $245^{*}$ & 2.0 & & 275 & 2.0 & & 220 & 2.0 & & 190 & 2.0 & \\
\hline
\end{tabular}

قيمـت الفعاليـة الحيويـة لليكندات والمعقدات ضــــات البكتريـا المرضـية Escherichia.coli و

Pseudomonas aeruginosa Staphylococcus aureus و ولوحظ ان لمعظم المركبات فعَاليّة جيدة ضد البكتريا الثلاثة ما عدا البكتريا Klipsila.Pneumonia فلم تستطع المركبات تتبيطها , وقد لوحظ أنَّ المعقدات تمتلك فعالية اكثرمن الليكندات، إذ أن المعقدات تحتوي على فلزات تتناسق مع مجاميع SH حيث ترتبط هذه الذرات مع خلايا الانزيم فتعمل بشكل اقوى من الذرات المانحة في الليكندات والتي جاءت متفقة مـع ما هومنشور (25)(49,48). والنتائج

$$
\text { موضحة في الجدول (12,11,10) والصور (3,2,1). }
$$

الجدول (10) فعّاليّة الليكندات كمُضادّات للبكتريا

\begin{tabular}{|c|c|c|c|c|c|c|c|c|c|c|c|c|c|c|c|c|}
\hline \multicolumn{4}{|c|}{ K.pnnemonia } & \multicolumn{4}{|c|}{ E.coli } & \multicolumn{4}{|c|}{ Staphylecocus oure } & \multicolumn{4}{|c|}{ Pseudomonas } & \multirow{2}{*}{ Sample } \\
\hline 62.5 & 125 & 250 & 500 & 62.5 & 125 & 250 & 500 & 62.5 & 125 & 250 & 500 & 62.5 & 125 & 250 & 500 & \\
\hline $\mathrm{R}$ & $\mathrm{R}$ & $\mathrm{R}$ & $\mathrm{R}$ & MS & MS & $\mathrm{S}$ & $\mathrm{S}$ & MS & MS & MS & $\mathrm{S}$ & $\mathrm{R}$ & $\mathrm{R}$ & $\mathrm{R}$ & MS & $\mathrm{T} 1 \mathrm{H}$ \\
\hline $\mathrm{R}$ & $\mathrm{R}$ & $\mathrm{R}$ & $\mathrm{R}$ & MS & MS & MS & $S$ & $\mathrm{~S}$ & $\mathrm{~S}$ & $\mathrm{~S}$ & $\mathrm{~S}$ & - & - & $\mathrm{R}$ & MS & $\mathrm{T} 2 \mathrm{H}$ \\
\hline $\mathrm{R}$ & $\mathrm{R}$ & $\mathrm{R}$ & $\mathrm{R}$ & $\mathrm{R}$ & MS & MS & MS & $\mathrm{S}$ & $\mathrm{S}$ & $\mathrm{S}$ & $\mathrm{S}$ & $\mathrm{R}$ & $\mathrm{R}$ & $\mathrm{R}$ & MS & L1H \\
\hline $\mathrm{R}$ & $\mathrm{R}$ & $\mathrm{R}$ & $\mathrm{R}$ & $\mathrm{R}$ & $\mathrm{R}$ & MS & MS & $\mathrm{S}$ & $\mathrm{S}$ & $\mathrm{S}$ & $\mathrm{R}$ & $\mathrm{R}$ & $\mathrm{R}$ & $\mathrm{R}$ & $\mathrm{S}$ & Mf \\
\hline $\mathrm{R}$ & $\mathrm{R}$ & $\mathrm{R}$ & $\mathrm{R}$ & MS & MS & MS & MS & $\mathrm{R}$ & $\mathrm{R}$ & MS & $\mathrm{S}$ & $\mathrm{R}$ & $\mathrm{R}$ & $\mathrm{R}$ & $\mathrm{R}$ & GH \\
\hline
\end{tabular}


الجدول (11) فعَاليّة المُعقّدات كمُضادّات للبكتريا

\begin{tabular}{|c|c|c|c|c|c|c|c|c|c|c|c|c|c|c|c|c|}
\hline \multicolumn{4}{|c|}{ K.pnnemonia } & \multicolumn{4}{|c|}{ E.coli } & \multicolumn{4}{|c|}{ Staphylecocus oure } & \multicolumn{4}{|c|}{ Pseudomona } & \multirow{2}{*}{ Sample } \\
\hline 62.5 & 125 & 250 & 500 & 62.5 & 125 & 250 & 500 & 62.5 & 125 & 250 & 500 & 62.5 & 125 & 250 & 500 & \\
\hline $\mathrm{R}$ & $\mathrm{R}$ & $\mathrm{R}$ & $\mathrm{R}$ & S & $\mathrm{S}$ & $\mathrm{S}$ & $\mathrm{S}$ & $\mathrm{S}$ & $\mathrm{S}$ & S & $\mathrm{S}$ & $\mathrm{R}$ & MS & MS & MS & 1. \\
\hline $\mathrm{R}$ & $\mathrm{R}$ & $\mathrm{R}$ & $\mathrm{R}$ & MS & MS & MS & $\mathrm{S}$ & $\mathrm{R}$ & $\mathrm{R}$ & MS & $\mathrm{S}$ & $\mathrm{R}$ & $\mathrm{R}$ & $\mathrm{R}$ & MS & 2. \\
\hline $\mathrm{R}$ & $\mathrm{R}$ & $\mathrm{R}$ & $\mathrm{R}$ & $\mathrm{R}$ & $\mathrm{R}$ & MS & MS & $\mathrm{S}$ & $S$ & $\mathrm{~S}$ & $\mathrm{~S}$ & - & - & - & $\mathrm{R}$ & 3. \\
\hline $\mathrm{R}$ & $\mathrm{R}$ & $\mathrm{R}$ & $\mathrm{R}$ & $S$ & $\mathrm{~S}$ & $\mathrm{~S}$ & $\mathrm{~S}$ & $\mathrm{~S}$ & $\mathrm{~S}$ & $\mathrm{~S}$ & $\mathrm{~S}$ & $\mathrm{R}$ & $\mathrm{R}$ & $\mathrm{R}$ & MS & 4. \\
\hline $\mathrm{R}$ & $\mathrm{R}$ & $\mathrm{R}$ & $\mathrm{R}$ & $\mathrm{R}$ & MS & MS & MS & $S$ & $\mathrm{~S}$ & $\mathrm{~S}$ & $\mathrm{~S}$ & $\mathrm{R}$ & MS & $\mathrm{S}$ & $\mathrm{S}$ & 5. \\
\hline $\mathrm{R}$ & $\mathrm{R}$ & $\mathrm{R}$ & $\mathrm{R}$ & MS & MS & $\mathrm{S}$ & $\mathrm{S}$ & S & S & S & $\mathrm{S}$ & MS & MS & $\mathrm{S}$ & $\mathrm{S}$ & 6. \\
\hline $\mathrm{R}$ & $\mathrm{R}$ & $\mathrm{R}$ & $\mathrm{R}$ & $\mathrm{R}$ & $\mathrm{R}$ & MS & MS & $\mathrm{S}$ & $\mathrm{S}$ & $\mathrm{S}$ & $\mathrm{S}$ & $\mathrm{R}$ & $\mathrm{R}$ & $\mathrm{R}$ & $\mathrm{R}$ & 7. \\
\hline $\mathrm{R}$ & $\mathrm{R}$ & $\mathrm{R}$ & $\mathrm{R}$ & MS & $\mathrm{S}$ & $\mathrm{S}$ & $\mathrm{S}$ & MS & MS & MS & MS & - & $\mathrm{R}$ & $\mathrm{R}$ & $\mathrm{R}$ & 8. \\
\hline
\end{tabular}

الجدول (12) أقل تركيز تثبيطي من الليكندات

\begin{tabular}{|c|c|c|c|c|c|c|c|c|c|c|c|c|c|c|c|c|}
\hline \multicolumn{4}{|c|}{ K.pnnemonia } & \multicolumn{4}{|c|}{ Staphylecocus oureu } & \multicolumn{4}{c|}{ Pseudomonas } & \multirow{2}{*}{ Sample } \\
\hline $\mathbf{6 2 . 5}$ & $\mathbf{1 2 5}$ & $\mathbf{2 5 0}$ & $\mathbf{5 0 0}$ & $\mathbf{6 2 . 5}$ & $\mathbf{1 2 5}$ & $\mathbf{2 5 0}$ & $\mathbf{5 0 0}$ & $\mathbf{6 2 . 5}$ & $\mathbf{1 2 5}$ & $\mathbf{2 5 0}$ & $\mathbf{5 0 0}$ & $\mathbf{6 2 . 5}$ & $\mathbf{1 2 5}$ & $\mathbf{2 5 0}$ & $\mathbf{5 0 0}$ & \\
\hline- & - & - & - & 9 & 12 & 14 & 14 & 13 & 14 & 14 & 15 & 8 & 9 & 10 & 12 & $\mathrm{~T} 1 \mathrm{H}$ \\
\hline- & - & - & - & 12 & 14 & 14 & 16 & 15 & 15 & 15 & 15 & - & - & 7 & 11 & T2H \\
\hline- & - & - & - & 10 & 13 & 13 & 13 & 16 & 16 & 16 & 19 & 7 & 7 & 10 & 13 & L1H \\
\hline- & - & - & - & 9 & 12 & 12 & 15 & 14 & 14 & 16 & 16 & 7 & 10 & 11 & 15 & L2H \\
\hline- & - & - & - & 10 & 10 & 12 & 14 & 15 & 18 & 18 & 18 & 8 & 10 & 10 & 15 & MF \\
\hline- & - & - & - & 12 & 12 & 14 & 14 & 10 & 10 & 11 & 15 & 8 & 8 & 8 & 8 & GH \\
\hline 21 & - & - & - & 21 & - & - & - & 21 & - & - & - & 21 & - & - & - & Cipr \\
\hline
\end{tabular}

الجدول(13) أقل تركيز تثبيطي من والمُعقّدات

\begin{tabular}{|c|c|c|c|c|c|c|c|c|c|c|c|c|c|c|c|c|}
\hline \multicolumn{4}{|c|}{ K.pnnemonia } & \multicolumn{4}{|c|}{ E.coli } & \multicolumn{4}{|c|}{ Staphylecocus oure } & \multicolumn{4}{|c|}{ Pseudomonas } & \multirow{2}{*}{$\begin{array}{c}S a \\
m\end{array}$} \\
\hline 62.5 & 125 & 250 & 500 & 62.5 & 125 & 250 & 500 & 62.5 & 125 & 250 & 500 & 62.5 & 125 & 250 & 500 & \\
\hline- & - & - & - & 15 & 15 & 18 & 20 & 14 & 15 & 15 & 16 & 8 & 9 & 10 & 10 & 1. \\
\hline- & - & - & - & 12 & 12 & 12 & 15 & 18 & 9 & 12 & 15 & 9 & 10 & 10 & 14 & 2. \\
\hline- & - & - & - & 10 & 10 & 11 & 11 & 16 & 16 & 18 & 18 & - & - & - & 8 & 3. \\
\hline- & - & - & - & 18 & 19 & 20 & 20 & 15 & 16 & 19 & 20 & 8 & 10 & 10 & 14 & 4. \\
\hline- & - & - & - & 12 & 13 & 13 & 14 & 16 & 18 & 20 & 21 & 25 & 28 & 30 & 35 & 5. \\
\hline- & - & - & - & 10 & 12 & 13 & 14 & 17 & 18 & 20 & 21 & 10 & 11 & 15 & 17 & 6. \\
\hline- & - & - & - & 10 & 13 & 14 & 14 & 15 & 16 & 16 & 16 & 9 & 7 & 7 & 7 & 7. \\
\hline- & - & - & - & 13 & 16 & 18 & 19 & 11 & 11 & 12 & 12 & - & 8 & 8 & 8 & 8. \\
\hline- & - & - & - & 21 & - & - & - & 21 & - & - & - & 21 & - & - & Cipr. & 9. \\
\hline
\end{tabular}

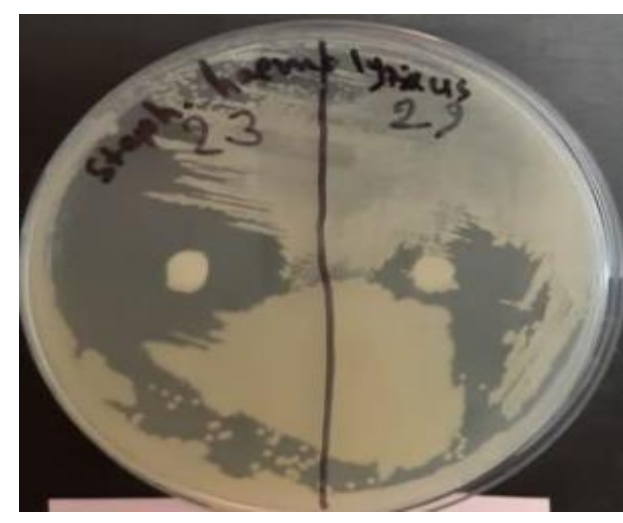

الصورة (3)تثبيط المعقد (6) لبكتريا Staphylococcus aureus

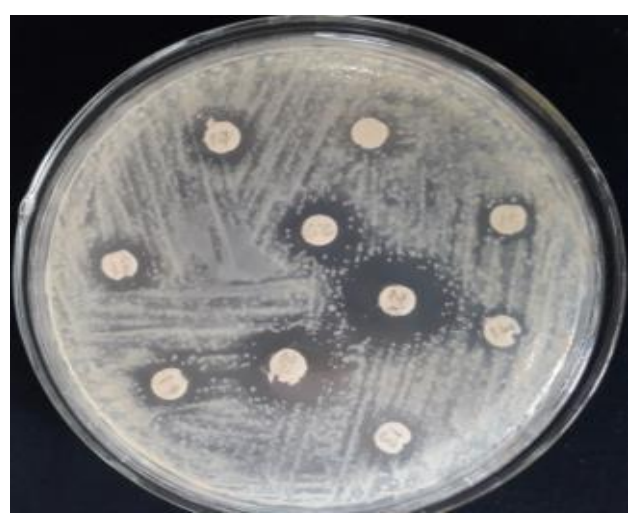

E.coli الصورة (2)تثبيط المعقد(4) البكتريا

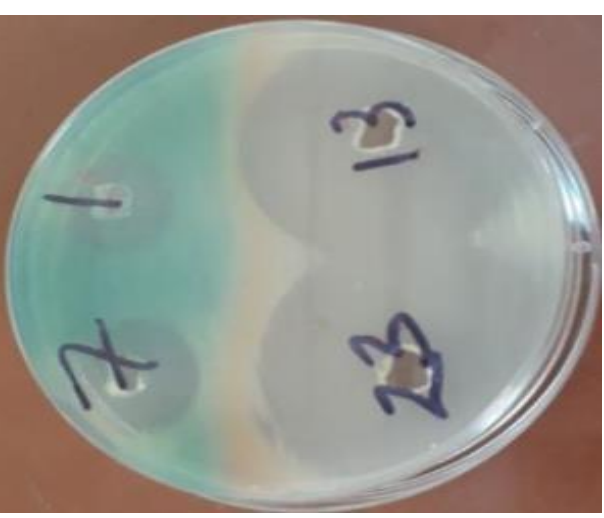

الصورة (1) تثبيط المعقد (5) لبكتريا

Pseudomonas arecnosa 
الاستتتاجات

إعتماداً على النتائج التي ظهرت والدراسات التحليليّة والفيزيائيّة والفعّاليّة البيولوجية أُستتتج الاتي:

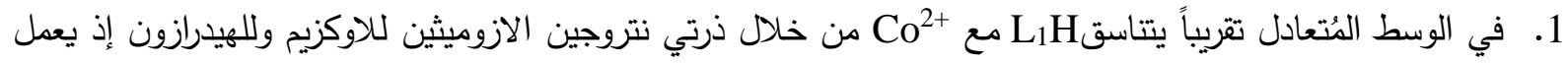
الليكند بشكل متعادل ثنائي السن كيليتي، اما في الوسط القاعدي فيتناسق بشكل سالب الثحنة ثنائي السن كيليتي

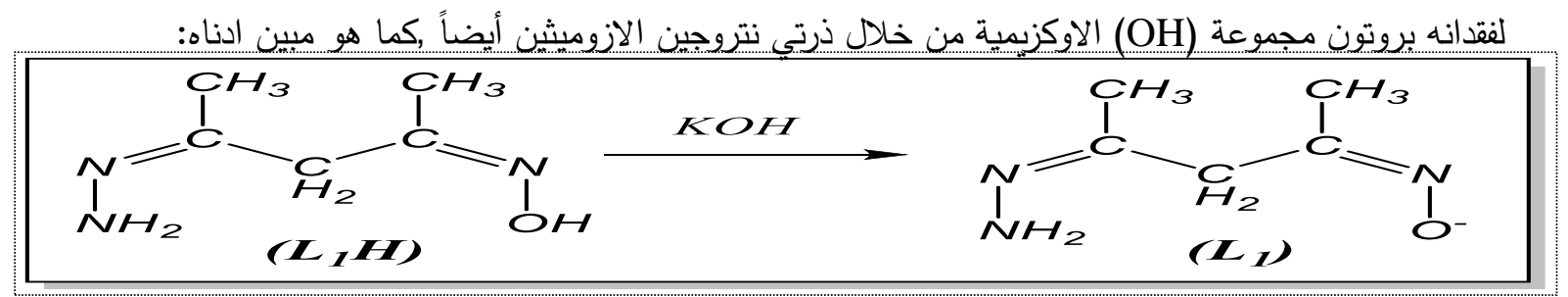

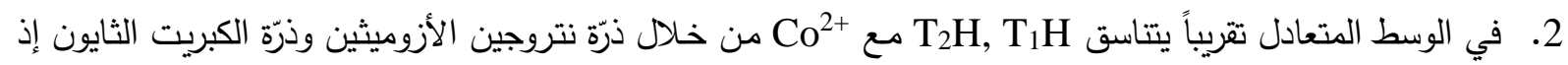
يعملان بشكل ليكند ثنائي السن متعادلي الثحنة، اما في الوسط القاعدي فيتاسقان من خلال ذرة نتروجين الازوميثين

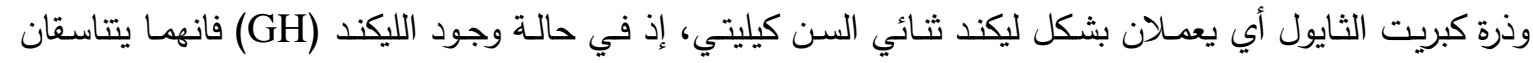
بشكل (Co-SH) متعـادلي الثحنة، أمّا في حالة وجود الليكند (mf) فانهما يفقدان بروتون ويصبحان مشحونين بشحنة سالبة ويتاسقان بشكل (Co-S') - وكما مبين ادناه.
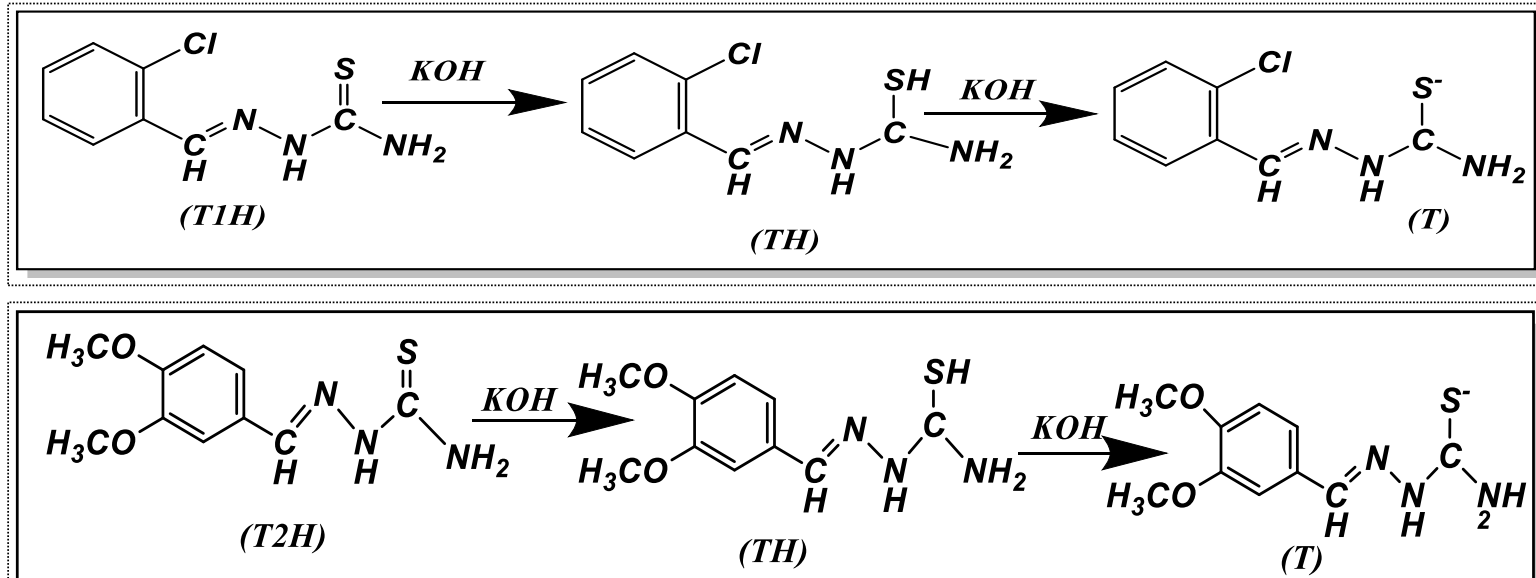

3. يتتاسق mf مع إذ في كل من الوسطين المتعادل والقاعدي من خلال ذرتي نتروجين مجموعتي (C=N) فيعل بشكل ليكند متعادل ثنائي السن كيليتي.

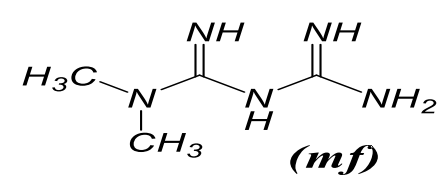




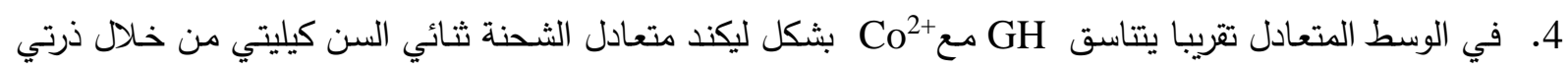
النتروجين والاوكسجين، اما في الوسط القاعدي فيعمل بشكل ليكند سالب الثحنة ثنائي السن كيليتي (بسبب فقدانه بروتون) من خلال ذرتي النتروجين والاوكسجين وكمامبين ادناه.

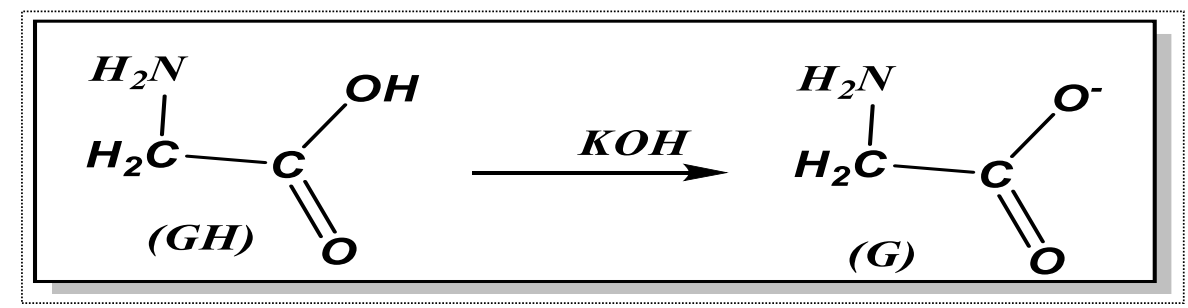

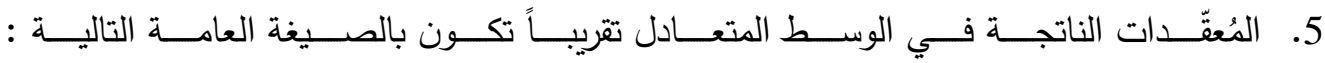

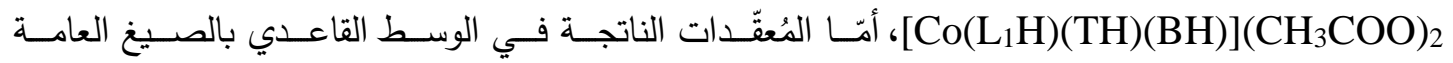

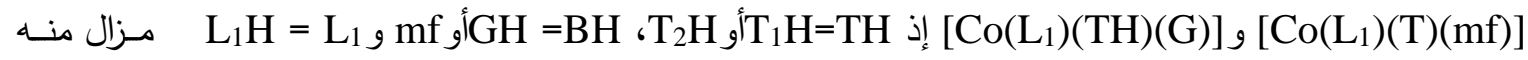
مزال منه بروتون).

6. يكون +Co في جميع المعقدات سداسي التتاسق ويكون شكل المعقدات ثماني السطوح من إن الليكندات والمُعقّدات
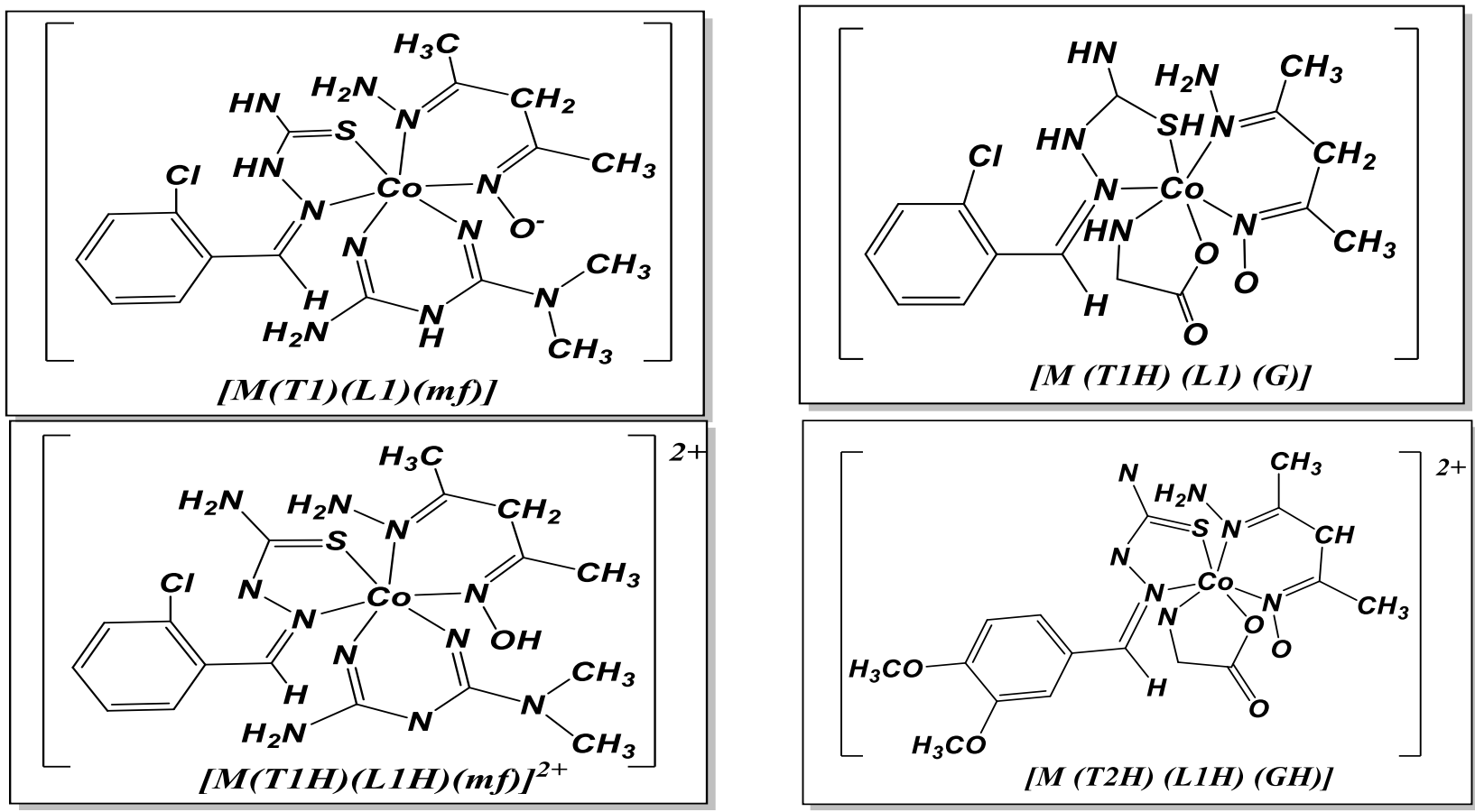

7. من خلال دراسة تاثير الليزر على الليكندات والمعقدات وجد ان المعقدات مستقرة تجاهها .

8. تبين انها قادرة على تثبيط بكتريا Escherichia.coli و و Stipsila pnnemoia و و Pseudomonas aeruginosa افضل من الليكندات وان بعض المعقدات تمتلك كفاءة تثبيط أعلى من المضاد الحيوي. 


\section{المصادر}

1. A. Stewertka, Aguide to the elements, Oxford Univ. Press Inc., New York, Rev. ed., 93, (1998).

2. G. Wilkinson, R.D. Gillard and J.A. McCleverty, Comprehensive coordination chemistry, Pergamon press, Oxford, England, Vol.4, 218-219, Vol 6, 584-684, (1987).

3. P. Chaudhary and Shelly, IJPSR, II (1): 39-47, (2011).

4. A.A. Yagani, G. Ozbolat and A. Tuli, Acta Biologica Turcica, 30(1): 22-26, (2018).

5. E.C. Mazarakoti, A.S. Beobide, V. Angelidou, C.G. Efthymiou, A. Terzis, V. Psycharis, G.A. Voyiatzis and S.P. Perlepes, Molecules, 24, 2219-2223, (2019).

6. S.R. Kelode, J. Chem. \& Pharm. Res., 5(6): 60-63, (2013).

7. M. Han, H. Bekci, A. Cumaoglu and S.G. Kucukguzei, Marmara pharm. J., 22(4): 559569, (2018).

8. B.A. Salah, A. Kandil and M.G. Abd El-Nasser, J. Rad. Res. \& Appl. Sci., 12(1): 383392, (2019).

9. F.R. Pavan, P.I. Das Maia, S.R. Leite, V.M. Deflon, A.A. Batista, D.N. Sato, S.G. Franzblau and C.Q. Leile, Eur. J. Med. Chem., 45(5): 1898-1905, (2010).

10. CH. Balachandran, J. Haribabu, K. Jayalakshmi, N.S.P. Bhuvanesh, R. Karvanbu, N. Emi and S. Awale, J. Inorg. Biochem., 182, 208-221, (2018).

11. M.A. Hussein, M.A. Iqbal, M.I. Umar, R.A. Haque and T.S. Guna, Aradian J. Chem., 12(8): 3183-3192, (2019).

12. S. Savir, Z.J. Wei, J.W.K. Liew, I. Vythilingam, Y.A. Lim, H.M. Saad, K.S. Sim and K.W. Tan, J. Mol. Str., 1211, 128090,(2020).

13. L. Logie, J. Harthill, K. Patel, S. Bacon, D.L. Hamithon, K. Macrae, G. McDoygall, H.H. Wang, L. Xue, H. Jiang, K. Sakamoto, A.R. Prescott and G. Rena, Diabetes, 61(6): 14231433, (2012).

14. H. Zaharenko, Pharmacogenetics of efficiency and tolerance of the perpral antdiabetic drug metformin, Ph.D. Thesis, Molecular Biology, Lativa Univ., 18-22, 48-50, (2015).

15. D. Vasak, N. Smrecki, B. Prugsvecki, I. Dilovic, I. Kirasic, D. Zilic, S. Moratovic and D. Matkovic- Calogovic, RSC adv., 9, 21637-21645, (2019).

16. B.I. Al-Abdali, I.M.A. Shakir and K.M. Nafea, Iraq, J. Sci., 65(4B): 3036-3047, (2015).

17. H.B. Shawish, M. Maah, S.N. Abdul Halim and Sh.A. Shaker, Irabian J. Chem., 9(2): 81935- 81942, (2016).

18. P. Vasantha, B.S. Kumar, B. Shekhar and P.V.A. Lakshmi, Mat. Sci. \& Eng., C, 90(1): 621- 633, (2018).

19. O.F. Akinyele, E.G. Fakola, L.M. Durosinmi, T.A. Ajayeoba and A.O. Ayeni, Lofe J. Sci., 21(3): 001, (2019).

20. A.I. Vogel, Textbook of practical organic chemistry, Longman Green, London, $3^{\text {rd }}$ Ed., 344, (1964).

21. Mahto B.C. (1981), "Studies on the complexes of 3-hydroxy-2- naphthaldehyde thiosemicarbazone with some divalent and trivalent metal ions", J. Indian Chem. Soc., 8, 935.

22. A.I. Vogel, "Textbook of quantitative inorganic analysis", Longman Inc., New York, $4^{\text {th }}$ Ed., 379-380, (1981).

23. A.M. James and F.E. Prichard, Practical physical chemistry", $3^{\text {rd }}$ Ed., Translated by M.S. Said, N.S.H. Al-Chalabi and L.S.M. Al-Jumard, Mosul Univ., (1995).

24. C.H. Collins, P.M. Lyne and J.M. Grange, Microbiological Methods, Buterwoths, London, $6^{\text {th }}$ Ed., 409, (1989).

25. A. Kshirsagar, M.P. Toraskar, V.M. Kukarn, S. Dhanashire and V. Kadam, Int. J. Chem. Tech. Res., 1(3): 696-701, (2009).

26. Y. Li and J. Zhang, Chin. Med. J. (Engl), 113(10): 948-956, (2000). 
27. D.L. Nelson and M.M. Cax, Principle of Biochemistry, W.H. Freeman, New York, 27, 675-677, 844, 854, (2005).

28. W.J. Geary, Cood. Chem. Rev., 7, 81, (1981).

29. M.N.O. Sadika, Elements and electromagnetics, Oxford Univ. Press., New York, $4^{\text {th }}$, ed., 386, (2007).

30. D.J. Griffiths, Introduction to electrodynamics, Peurson Education Inc., $4^{\text {th }}$ Ed., 312-332, (2013).

31. A.B.P. Lever, J. Chem. Edu., 45, 711-712, (1968).

32. N.M.H. Salem, L. El-Sayed, W. Hazse and M.F. Iskander, J. Coord. Chem., 68(12): 21472166, (2015).

33. R. Chaudhary and Shelly, IJPSR, II (1): 39-47, (2011).

34. S. Deveci, J. Inc. Phenom., 77: 1-4, (2013).

35. C. Dometgul, Synthesis and characterization of oxime compounds immobilized to solid support and their metal complexes, Ph. D. Thesis, Chem. Dept., Institute \& Natural and Applied Sci., Cukurova Univ., (2008).

36. K. Nakamoto, Infrared and raman spectra of inorganic and coordination compound", John Wily and Sons, New York, $3^{\text {rd }}$ Ed., 166-322, (1976).

37. A.M.A. Al-Izzy, Preparation and characterization of some new zinc (II) complexes with mixed ligands and study of laser effect on them, M. Sc. Thesis, Mosul Univ., Educ. College, Mosul -Iraq, 45-46, (2013).

38. E. Pahontu, V. Fala, A. Gulea, D. Poiri, V. Tapcov and T. Rosu, Molecules, 18, 88128836, (2013).

39. P.K. Singh, J.K. Koacher and J.P. Tandon, J. Inorg. Nucl. Chem., 43, 1775-1778, (1981).

40. I. Suzuki, Bull. Chem. Soc., Japan, 35, 1449, (1962).

41. S. Priyarega, P. Kalaivani, R. Prabhakaran, T. Hashimoto, A. Endo and K. Natarajan, J. Mol. struct., 1002(1): 58-62, (2011).

42. M.W.I. Ali, Preparation of cobalt (II) and nickel (II) complexes containing mixes ligands and their radiolytical study, M.Sc. Thesis. Dept., Education Coll., Mosul Univ., MosulIraq, (2002).

43. N. Kanoongo, R. Singh and J.P. Tandon, Met org. Chem., 17( 8-9): 837-847, (1987).

44. O.R. Bada, M. Marinesen, D. Chifirln, ano M. Beleotu, Eur. J. Med. Chem., 45 (7): 3027 3034, (2010).

45. G. Patrinoiu, L. Patron, O. Carp and N. Stanica, J. Therm. Anal. Color., 72 (2): 484-495, (2003).

46. .P. O'Brien , , J. Chem. Edu., 59, 1052, (1982).

47. V.K. Saxena, Gupta M. and M.N. Srivastava, Synth. React. Inorg. \& Met.- org. Chem., 26(10): 1661-1676, (1996).

48. H.M. Vinusha, S.P. Kollur, R. Ramu, P.S. Shirahathi, N.M.N. Parasad and M. Begum, Lett. Appl. Nano-Bio. Sci., 9(3):1372-1388, (2020).

49. E. Jawetz, J.I. Melnick and E.A. Adelberg, Review of medical microbiology, Middle East Ed., Appleton \& Lamage Libieividu Liban, $17^{\text {th }}$ Ed., 71-73, 136, (1983).

50. N. Csele, Fundamentals of light sources and laser, John Wiley and Sons, Ch. 9, (2004).

51. C.S. Willet, An introduction to gas laser", Pergamon Press, 407-411, (1974).

52. S. Farivar, T. Malekshahabim and R. Shiariy, J. Lasers Med. Sci., 5(2): 52-62, (2014). 